\title{
Effective Field Theory for Bound State Reflection
}

\author{
Michelle Pine and Dean Lee \\ North Carolina State University
}

\begin{abstract}
Elastic quantum bound-state reflection from a hard-wall boundary provides direct information regarding the structure and compressibility of quantum bound states. We discuss elastic quantum bound-state reflection and derive a general theory for elastic reflection of shallow dimers from hard-wall surfaces using effective field theory. We show that there is a small expansion parameter for analytic calculations of the reflection scattering length. We present a calculation up to second order in the effective Hamiltonian in one, two, and three dimensions. We also provide numerical lattice results for all three cases as a comparison with our effective field theory results. Finally, we provide an analysis of the compressibility of the alpha particle confined to a cubic lattice with vanishing Dirichlet boundaries.
\end{abstract}




\section{Contents}

I. Introduction 3

II. Formalism

A. General Two-body Bound States 6

B. Shallow Dimer Reflection 7

1. The Effective Hamiltonian 7

III. Results

A. Results in One Dimension 14

1. First Order Effective Hamiltonian in One Dimension 14

2. Second Order Effective Hamiltonian in One Dimension 14

B. Results in Two Dimensions

1. First Order Effective Hamiltonian in Two Dimensions 16

2. Second Order Effective Hamiltonian in Two Dimensions 16

C. Results in Three Dimensions 17

1. First Order Effective Hamiltonian in Three Dimensions 17

2. Second Order Effective Hamiltonian in Three Dimensions 18

IV. Scattering Length and Reflection Radius 18

A. Numerical Results

1. Numerical Results in One Dimension 20

2. Numerical Results in Two Dimensions 22

3. Numerical Results in Three Dimensions 23

4. Alpha Particle 25

V. Summary and Future Work 27

References

A. Derivation of the Diagonal Adiabatic Correction 29

B. Diagonal Adiabatic Correction in One Dimension 32 


\section{INTRODUCTION}

The study of confined quantum bound states has interesting applications in many areas including nuclear structure calculations, experimental ultracold atomic physics, and quantum dots and wells. Elastic reflection off of a hard wall allows one to gain insight into the structure of a bound state as well as how it behaves when confined or compressed. In the case of quantum dots and wells, the bound state is comprised of an electron and hole confined inside a two- or three- dimensional nanoscale semiconductor structure. Varying the geometry of the structure allows increased control of current tunneling as well as photon absorption and emission. See for example Ref. [1 [4]. The theory developed in this paper gives a universal result for the energy of Wannier excitons in direct-band gap semiconductors as a function of the binding energy, effective masses, and the geometry of the nanostructure.

In the case of ultra-cold atomic experiments, a quantum well is produced by using lasers to create repulsive boundaries, thereby confining the atoms on an optical lattice. Similar ideas have been proposed for quantum billiards systems [5]. Since they add no dimensionful scale to the problem, hard-wall boundaries provide a probe of universal physics at large scattering lengths. For example the scattering length for particle-particle scattering is directly proportional to the scattering length for dimer-wall reflection. In this paper we calculate this universal proportionality constant in one, two and three spatial dimensions.

It is not possible, experimentally, to construct a hard-wall boundary for protons and neutrons, so it may seem that the current discussion has no direct connection with nuclear physics. A similar critique could be made of Lüscher's analysis of periodic boundaries in finite cubic volumes [6, 7]. However, Lüscher's analysis now provides the theoretical framework for numerous calculations in lattice quantum chromodynamics [8-10]. While it is not possible to construct a hard-wall boundary for protons and neutrons in nuclear physics experiments, it is possible to do so in ab initio numerical calculations. In some cases such calculations can be used to verify phenomena observed in experiments, and in other cases the calculations probe 
new physics inaccessible in the laboratory. As with temperature and chemical potential, boundaries can provide tunable control parameters for use in such calculations. Hard-wall boundaries can and have been incorporated into ab initio numerical calculations [11, 12]. Hard-wall boundaries are currently being used to perform nuclear lattice calculations that probe properties such as structure and elastic deformation of nuclei. The method is a useful compliment to Luscher's periodic boundary analysis for bound states and scattering states. By varying the boundary conditions one can calculate quantities such as nuclear radii and quantities analogous to bulk and shear modulus, which can then be compared with bulk modulus estimates inferred from observed monopole resonance energies.

Recently there has been a great deal of interest in alpha-particle clusters confined inside nuclei such as carbon-12 [13-15]. Very recently ab initio lattice effective field theory calculations of carbon-12 have given the energies of the ground state, the excited spin-2 state, and, for the first time, the Hoyle state responsible for the formation of carbon in stellar environments [16]. Furthermore, these calculations indicate the presence of compressed, correlated alpha clusters. Since there are no low-energy resonances of the alpha particle and little experimental data on the compression of alpha particles, a better understanding of this phenomenon would prove valuable. In this paper we analyze the compressibility of the alpha particle.

In this paper we discuss the elastic reflection of quantum bound states off of hard-wall boundaries. We begin with a short presentation of the general case, where the number of dimensions and number of constituent particles is completely arbitrary. We then develop a general theory from the principles of effective field theory for the specific case of shallow two-body quantum bound state reflection in one, two, and three dimensions. Our main result is a derivation of the phase shift due to the scattering of a two-particle bound state on a hard wall in the adiabatic limit and up to second order in an expansion of the effective Hamiltonian. The effective field theory results are presented for the cases of one, two, and three dimensions for arbitrary mass ratio $m_{2} / m_{1}$ and compared to numerical results. Finally, we provide an analysis of the compressibility of the alpha particle, summarize, and consider further work to be done. A partial summary of our results was presented in a previous letter publication [17]. Here we present the full details of our calculations. We note that very recently there has been work on two body systems in a finite volume with periodic boundaries [18, 19] as well as two body systems with Neumann boundary conditions [20]. 


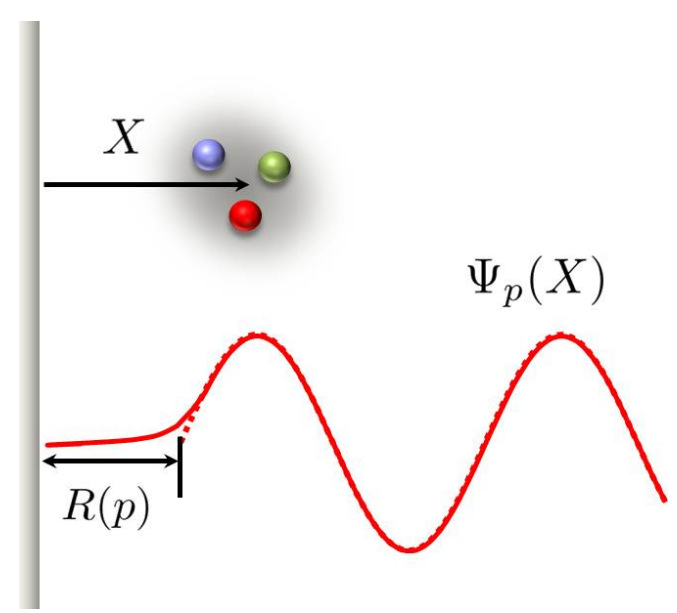

FIG. 1: Sketch showing the distance X from the wall to the center of mass of the bound state and the standing wave solution

\section{FORMALISM}

We consider a non-relativistic bound state in $d$ dimensions, with total mass $M$, and arbitrary number of constituent particles. The bound state scatters elastically off of a hardwall boundary, implemented as a vanishing Dirichlet boundary condition. We let $X$ be the distance from the wall to the center of mass of the bound state. We work in the inertial frame where the only non-zero component of the center-of-mass momentum is the component perpendicular to the hard-wall boundary. Then we construct a standing wave solution with center-of-mass momenta $\pm p$ perpendicular to the wall with reflection phase shift, $\delta(p)$, and reflection radius, $R(p)=-\delta(p) / p$. The reflection radius is a measure of the distance between the wall and the closest node of the asymptotic standing wave, $\Psi_{p}(X) \propto \sin [p X+\delta(p)]$. This is shown in Fig. 1.

Reflection in one dimension is analogous to $S$-wave scattering in three dimensions and so we use the effective range expansion,

$$
p \cot \delta(p)=-\frac{1}{a_{R}}+\frac{1}{2} r_{R} p^{2}-\mathcal{P}_{R} p^{4}+\cdots,
$$

where $a_{R}$ is the scattering length, $r_{R}$ is the effective range, and $\mathcal{P}_{R}$ is the shape parameter. Notice that at threshold, $a_{R}$ is equal to the reflection radius, $a_{R}=\lim _{p \rightarrow 0} R(p)$. For a completely rigid bound state, $R(p)=a_{R}$ for all $p$. However, we expect that the bound state will not be perfectly rigid and so it will compress more when the collision energy increases. This means that the reflection radius, $R(p)$, will decrease with increasing bound state center- 
of-mass momentum, $p$. The rate of decrease measures the compressibility of the bound state under unilateral stress.

We now consider the bound state confined to a $d$-dimensional cubic box with length $L$ and hard wall boundaries on all sides. In the limit of large $L$, we can approximate the wavefunction as a product of standing waves along each coordinate axis. Each standing wave has half-wavelength equal to $L-2 R[p(L)]$, and so the reflection radius can be related to the ground state confinement energy of the bound state as follows:

$$
E(L)=\frac{p^{2}(L) \cdot d}{2 M}\left[1+O\left(L^{-2}\right)\right]
$$

where

$$
p(L)=\frac{\pi}{L-2 R[p(L)]} .
$$

This relation can be used to determine the reflection radius as a function of the centerof-mass momentum. The $O\left(L^{-2}\right)$ relative error in Eq. (2) arises because the different coordinates for $d>1$ cannot be exactly separated as a product of standing waves. This is due to the effects of double-wall collision near the wall intersections. By wall intersections we mean the corners of a two-dimensional square or the edges of a three-dimensional cube. In each case, the codimension of the wall intersections is two, and this explains the $O\left(L^{-2}\right)$ relative error. In one dimension, however, there are no wall intersections, and so the error is exponentially small in $L$.

\section{A. General Two-body Bound States}

We now consider the more specific case of a bound state with zero orbital angular momentum consisting of two distinguishable particles with masses $m_{1}$ and $m_{2}$. The reduced mass, $\mu$, is defined in the usual way, $\mu=\left(m_{1}^{-1}+m_{2}^{-1}\right)^{-1}$. We let $E_{B}$ be the infinite volume binding energy of the dimer, and $\kappa_{B}$ be the binding momentum defined by the relation $E_{B}=-\kappa_{B}^{2} /(2 \mu) . \quad a_{B}$ is the $S$-wave scattering length for shallow-binding particle-particle scattering. In the shallow-binding limit, where $a_{B}$ is much larger than the range of the interaction $\kappa_{B}=a_{B}^{-1}$, we can neglect the short-distance physics. So, the reflection phase shift is a universal function of the dimensionless ratio $p / \kappa_{B}$. In this paper we present the form of this universal function. 


\section{B. Shallow Dimer Reflection}

Next we use the principles of effective field theory to calculate the reflection scattering length for a shallow dimer. While effective field theory is a well established method, in the current case we are dealing with a non-homogenous system for which there is no immediately obvious small expansion parameter. In the soft scattering limit, we use an adiabatic approximation for the center-of-mass motion of the system. We then perform an asymptotic expansion to account for the long-distance physics. The result is an expansion for $\kappa_{B} a_{R}$ with convergence controlled by an expansion parameter $e^{-2 \kappa_{B} a_{R}}$.

We let $\vec{r}_{1}$ and $\vec{r}_{2}$ be the coordinates of the two constituent particles and assume an attractive short-range interaction given by the Hamiltonian,

$$
H=-\frac{1}{2 m_{1}} \vec{\nabla}_{r_{1}}^{2}-\frac{1}{2 m_{2}} \vec{\nabla}_{r_{2}}^{2}+C_{B} \bar{\delta}^{(d)}\left(\vec{r}_{1}-\vec{r}_{2}\right)
$$

where $\bar{\delta}^{(d)}$ is a regulated $d$-dimensional delta function. The coefficient $C_{B}$ is tuned to produce a bound state with energy $E_{B}$ at infinite volume. We take $\vec{r}=\vec{r}_{1}-\vec{r}_{2}$ to be the relative separation between the particles. For any fixed center-of-mass coordinate, the part of the Hamiltonian that is only dependent on the relative coordinate, $\vec{r}$, is given by

$$
H_{\mathrm{rel}}=-\frac{1}{2 \mu} \vec{\nabla}_{r}^{2}+C_{B} \bar{\delta}^{(d)}(\vec{r})
$$

\section{The Effective Hamiltonian}

Let $E_{K}$ be the kinetic energy of the moving dimer. To calculate the reflection scattering length it suffices to consider dimer-wall scattering in the limit $E_{K} \ll\left|E_{B}\right|$. In this low-energy limit we perform an adiabatic expansion for the center-of-mass motion. This technique is conceptually similar to the adiabatic hyperspherical approximation [21 23].

We let $X$ be the distance from the wall to the center of mass of the dimer. We label the coordinate axes $1,2, \ldots, d$, and take the $d^{\text {th }}$ dimension to be perpendicular to the wall. For fixed $X$ the hard-wall boundary at $\left(r_{1}\right)_{d}=0$ gives a minimum value for the relative coordinate $r_{d}$,

$$
r_{d}^{\min }=-\frac{M}{m_{2}} X
$$


We now define $x_{-}$as twice this distance,

$$
x_{-}(X)=-\frac{2 M}{m_{2}} X
$$

So $x_{-}(X)$ is twice the distance from the wall to $\vec{r}_{2}$ when $\vec{r}_{1}$ touches the wall. In other words $\psi_{X}(\vec{r})$ must vanish when $\vec{r}_{d}$ equals $-x_{-}(X) / 2$. The factor of two simplifies the expansion to be derived later. Similarly the hard-wall boundary for $\vec{r}_{2}$ gives a maximum value for $r_{d}$,

$$
r_{d}^{\max }=\frac{M}{m_{1}} X
$$

As in the previous case, we define

$$
x_{+}(X)=\frac{2 M}{m_{1}} X
$$

So $x_{+}(X)$ is twice the distance from the wall to $\vec{r}_{1}$ when $\vec{r}_{2}$ touches the wall. In other words $\psi_{X}(\vec{r})$ must vanish when $\vec{r}_{d}$ equals $x_{+}(X) / 2$. Once again the factor of two has been added to simplify the expansion to be derived later.

We now define the $d$-dimensional vectors

$$
\begin{aligned}
& \vec{r}_{-}(X)=\left(0, \cdots, 0, x_{-}(X)\right), \\
& \vec{r}_{+}(X)=\left(0, \cdots, 0, x_{+}(X)\right) .
\end{aligned}
$$

The magnitudes of the vectors are

$$
\begin{gathered}
r_{-}(X)=\left|\vec{r}_{-}(X)\right|=-x_{-}(X), \\
r_{+}(X)=\left|\vec{r}_{+}(X)\right|=x_{+}(X) .
\end{gathered}
$$

Let $E_{\kappa}$ be the kinetic energy of the moving dimer. For each $X$ it is only necessary to keep the ground state of $H_{\text {rel }}$ satisfying the hard-wall boundary condition. This is due to the fact that the contribution of the excited states are suppressed by powers of $E_{K} /\left|E_{B}\right|$ and hence cannot contribute to the reflection scattering length, but only to higher-order coefficients in the effective range expansion.

For each $X$ we take $\psi_{X}(\vec{r})$ to be the normalized ground state wavefunction of $H_{\text {rel }}$ satisfying the hard-wall boundary constraint. Then the eigenstates of the full Hamiltonian are

$$
|\Psi(X)\rangle \otimes\left|\psi_{X}(\vec{r})\right\rangle+O\left(P^{2} / \kappa_{B}^{2}\right)
$$


where $P$ is the center-of-mass momentum of the dimer in the direction perpendicular to the wall. The low-energy effective Hamiltonian is

$$
H_{\mathrm{eff}}=-\frac{1}{2 M} \frac{\partial^{2}}{\partial X^{2}}+V(X)+T(X)
$$

where $V(X)$ is the adiabatic potential,

$$
V(X)=\left\langle\psi_{X}\left|H_{\mathrm{rel}}\right| \psi_{X}\right\rangle
$$

and $T(X)$ is the diagonal adiabatic correction, $T(X)=-\frac{1}{2 M}\left\langle\psi_{X}\left|\frac{\partial^{2}}{\partial X^{2}}\right| \psi_{X}\right\rangle$.

Notice that the cross-term resulting from one derivative with respect to $X$ vanishes because of the fixed normalization of $\psi_{X}$. We use lattice regularization to deal with the continuum limit singularity in the delta function for $d>1$.

We define the local $X$-dependent energy, $E_{\vec{r}_{-}, \vec{r}_{+}}$, and binding momentum, $\kappa_{\vec{r}_{-}, \vec{r}_{+},}$, in terms of the adiabatic potential

$$
V(X)=\left\langle\psi_{X}\left|H_{\mathrm{rel}}\right| \psi_{X}\right\rangle=E_{\vec{r}_{+}, \vec{r}_{-}}=-\frac{\kappa_{\vec{r}_{-}, \vec{r}_{+}}^{2}}{2 \mu},
$$

for the vanishing Dirichlet boundary condition. For large $X$ we can generate an asymptotic expansion for the local $X$-dependent binding momentum, $\kappa_{\vec{r}_{-}, \vec{r}_{+}}, V(X)$, and $T(X)$ in powers of $e^{-\kappa_{B} r_{+}(X)}$ and $e^{-\kappa_{B} r_{-}(X)}$. The boundary conditions are enforced by applying the method of images.

We consider two infinite periodic chains of delta functions. The first chain includes delta functions centered at multiples of $\vec{r}_{+}-\vec{r}_{-}$. The second chain includes a delta function centered at $\vec{r}_{+}$plus multiples of $\vec{r}_{+}-\vec{r}_{-}$. We now construct a wavefunction which is the lowest energy eigenstate for this potential with alternating signs at the centers of the neighboring delta functions. For a picture of the wavefunction see Fig. 2.

For this wavefunction we use an ansatz that is a superposition of $d$-dimensional Yukawa functions.

$$
\psi_{\vec{r}_{-}, \vec{r}_{+}}(\vec{r})=A_{\vec{r}_{-}, \vec{r}_{+}} \phi_{\vec{r}_{-}, \vec{r}_{+}}(\vec{r})
$$

where

$$
\begin{aligned}
\phi_{\vec{r}_{-}, \vec{r}_{+}}(\vec{r}) & =y_{d, 0}\left(\kappa_{\vec{r}_{-}, \vec{r}_{+}},|\vec{r}|\right)-y_{d, 0}\left(\kappa_{\vec{r}_{-}, \vec{r}_{+}},\left|\vec{r}-\vec{r}_{-}\right|\right)-y_{d, 0}\left(\kappa_{\vec{r}_{-}, \vec{r}_{+}},\left|\vec{r}-\vec{r}_{+}\right|\right) \\
& +y_{d, 0}\left(\kappa_{\vec{r}_{-}, \vec{r}_{+}},\left|\vec{r}-\vec{r}_{+}+\vec{r}_{-}\right|\right)+y_{d, 0}\left(\kappa_{\vec{r}_{-}, \vec{r}_{+}}, \vec{r}+\vec{r}_{+}-\vec{r}_{-}\right)+\cdots
\end{aligned}
$$




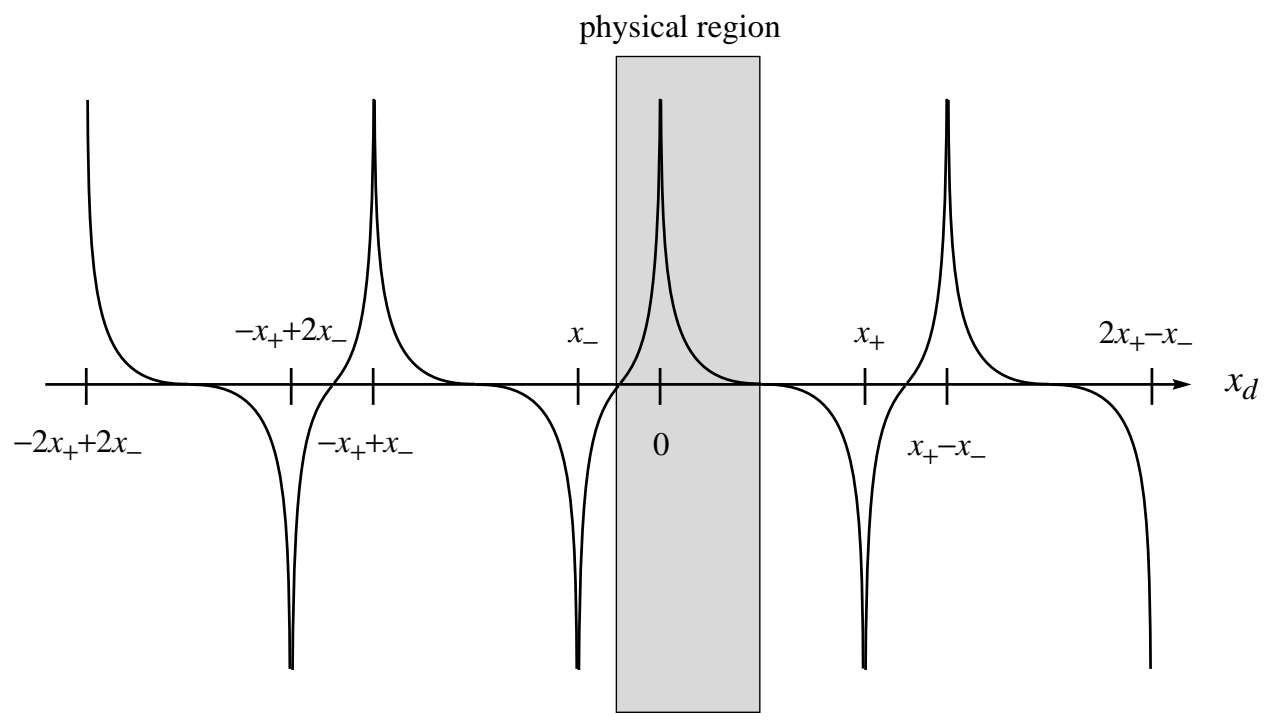

FIG. 2: Sketch of the wavefunction derived from the method of images

Here

$$
y_{d, 0}(\kappa, r)=\int \frac{d^{d} \vec{p}}{(2 \pi)^{d}} \frac{e^{-i \vec{p} \cdot \vec{r}}}{p^{2}+\kappa^{2}} .
$$

is the generalized $d$ dimensional Yukawa function, and we also define

$$
f_{d}\left(\kappa^{I}, \kappa^{I I}, r\right)=\int d^{d} \vec{r}^{\prime} y_{d, 0}\left(\kappa^{I},\left|\vec{r}^{\prime}\right|\right) y_{d, 0}\left(\kappa^{I I},\left|\vec{r}^{\prime}-\vec{r}\right|\right),
$$

as the full overlap integral of two Yukawa functions in $d$ dimensions whose centers are separated by a spatial distance $r$. In Eq.18) $A_{\vec{r}_{-}, \vec{r}_{+}}$is a function that normalizes $\psi_{\vec{r}_{-}, \vec{r}_{+}}(\vec{r})$, and we find that

$$
\begin{aligned}
& \int \frac{d^{d} \vec{p}}{(2 \pi)^{d}}\left[\frac{1}{\frac{p^{2}}{2 \mu}-E_{\vec{r}_{-}, \vec{r}_{+}}}-\frac{1}{\frac{p^{2}}{2 \mu}-E_{B}}\right] \\
& =\sum_{j} \int \frac{d^{d} \vec{k}}{(2 \pi)^{d}} \frac{e^{-i \vec{p} \cdot\left(-(j+1) r_{+}+j r_{-}\right)}}{\frac{p^{2}}{2 \mu}-E_{\vec{r}_{-}, \vec{r}_{+}}}-\sum_{j \neq 0} \int \frac{d^{d} \vec{k}}{(2 \pi)^{d}} \frac{e^{-i \vec{p} \cdot\left(-j r_{+}+j r_{-}\right)}}{\frac{p^{2}}{2 \mu}-E_{\vec{r}_{-}, \vec{r}_{+}}},
\end{aligned}
$$

where $j$ is summed over integer values.

At first order in powers of $e^{-\kappa_{B} r_{+}(X)}$ and $e^{-\kappa_{B} r_{-}(X)}$ we get a correction to the binding momentum

$$
\kappa_{\vec{r}_{-}, \vec{r}_{+}(1)}=\frac{\int \frac{d^{d} \vec{p}}{(2 \pi)^{d}}\left[\frac{e^{i \vec{p} \cdot \vec{r}_{+}}}{p^{2}+\kappa_{B}^{2}}+\frac{e^{i \vec{p} \cdot \vec{r}_{-}}}{p^{2}+\kappa_{B}^{2}}\right]}{\frac{\partial}{\partial \kappa_{B}}\left[\int \frac{d^{d} \vec{p}}{(2 \pi)^{d}} \frac{1}{p^{2}+\kappa_{B}^{2}}\right]} .
$$


The first-order correction to the adiabatic potential is

$$
V^{(1)}(X)=-\frac{\kappa_{B}}{\mu} \kappa_{\vec{r}_{-}, \vec{r}_{+}(1)}
$$

and the first order diagonal adiabatic correction is

$$
\begin{aligned}
T^{(1)}(X) & =\frac{M}{2 m_{1}^{2} f_{d}\left(\kappa_{B}, \kappa_{B}, 0\right)} y_{d, 0}\left(\kappa_{B}, r_{+}\right) \\
& +\frac{M}{2 m_{2}^{2} f_{d}\left(\kappa_{B}, \kappa_{B}, 0\right)} y_{d, 0}\left(\kappa_{B}, r_{-}\right) .
\end{aligned}
$$

So the first order correction to the effective Hamiltonian is

$$
\begin{aligned}
V^{1}(X)+T^{1}(X) & =-\frac{\kappa_{B}}{\mu} \kappa_{\vec{r}_{-}, \vec{r}_{+}(1)}+\frac{M}{2 m_{1}^{2} f_{d}\left(\kappa_{B}, \kappa_{B}, 0\right)} y_{d, 0}\left(\kappa_{B}, r_{+}\right) \\
& +\frac{M}{2 m_{2}^{2} f_{d}\left(\kappa_{B}, \kappa_{B}, 0\right)} y_{d, 0}\left(\kappa_{B}, r_{-}\right)
\end{aligned}
$$

At second order the correction to the local binding momentum is

$$
\begin{aligned}
& \kappa_{\vec{r}_{-}, \vec{r}_{+}(2)} \\
& =\frac{1}{\frac{\partial}{\partial \kappa_{B}} y_{d, 0}\left(\kappa_{B}, 0\right)}\left\{\frac{y_{d, 0}\left(\kappa_{B}, r_{+}\right)+y_{d, 0}\left(\kappa_{B}, r_{-}\right)}{\frac{\partial}{\partial \kappa_{B}} y_{d, 0}\left(\kappa_{B}, 0\right)} \frac{\partial}{\partial \kappa_{B}}\left[y_{d, 0}\left(\kappa_{B}, r_{+}\right)+y_{d, 0}\left(\kappa_{B}, r_{-}\right)\right]\right. \\
& \left.-2 y_{d, 0}\left(\kappa_{B}, r_{+}+r_{-}\right)-\frac{\left[y_{d, 0}\left(\kappa_{B}, r_{+}\right)+y_{d, 0}\left(\kappa_{B}, r_{-}\right)\right]^{2}}{2\left[\frac{\partial}{\partial \kappa_{B}} y_{d, 0}\left(\kappa_{B}, 0\right)\right]^{2}} \frac{\partial^{2}}{\partial \kappa_{B}^{2}} y_{d, 0}\left(\kappa_{B}, 0\right)\right\} .
\end{aligned}
$$

The correction to the adiabatic potential is

$$
V^{(2)}(X)=-\frac{\kappa_{B}}{\mu} \kappa_{\vec{r}_{-}, \vec{r}_{+}(2)}-\frac{1}{2 \mu} \kappa_{\vec{r}_{-}, \vec{r}_{+}(1)}^{2} .
$$

The diagonal adiabatic correction contains a number of terms which we unite as

$$
\begin{aligned}
T^{(2)}(X) & =T_{(A)}^{(2)}(X)+T_{(B)}^{(2)}(X)+T_{(C)}^{(2)}(X) \\
& +T_{(D 1)}^{(2)}+T_{(D 2)}^{(2)}(X)+T_{(D 3)}^{(2)}(X)+T_{(D 4)}^{(2)}(X)+T_{(E)}^{(2)}(X) .
\end{aligned}
$$

The first term in Eq. 29 is

$$
T_{(A)}^{(2)}(X)=-\frac{f_{d}\left(\kappa_{B}, \kappa_{B}, 0\right)}{2 M}\left(\frac{\partial A_{\vec{r}_{-}, \vec{r}_{+}(1)}}{\partial X}\right)^{2}+\cdots,
$$

where the ellipses indicate terms at third order or higher. Here 


$$
\begin{aligned}
A_{\vec{r}_{-}, \vec{r}_{+}} & =\left[f_{d}\left(\kappa_{\vec{r}_{-}, \vec{r}_{+}}, \kappa_{\vec{r}_{-}, \vec{r}_{+}}, 0\right)-f_{d}\left(\kappa_{\vec{r}_{-}, \vec{r}_{+}}, \kappa_{\vec{r}_{-}, \vec{r}_{+}}, r_{-}\right)-f_{d}\left(\kappa_{\vec{r}_{-}, \vec{r}_{+}}, \kappa_{\vec{r}_{-}, \vec{r}_{+}}, r_{+}\right)\right. \\
& \left.+2 f_{d}\left(\kappa_{\vec{r}_{-}, \vec{r}_{+}}, \kappa_{\vec{r}_{-}, \vec{r}_{+}}, r_{+}+r_{-}\right)+\cdots\right]^{-1 / 2}
\end{aligned}
$$

arises from the normalization of the relative coordinate wavefunction. Once again the ellipses indicate third order or higher terms. For more details see Appendix A. The derivative of the normalization function, at first order, with respect to $X$ is

$$
\begin{aligned}
& \frac{\partial A_{\vec{r}_{-}, \vec{r}_{+}(1)}}{\partial X} \\
& =\frac{1}{2} \frac{\frac{2 M}{m_{1}} \frac{\partial}{\partial r_{+}} f_{d}\left(\kappa_{B}, \kappa_{B}, r_{+}\right)+\frac{2 M}{m_{2}} \frac{\partial}{\partial r_{-}} f_{d}\left(\kappa_{B}, \kappa_{B}, r_{-}\right)-\frac{\partial \kappa_{\vec{r}_{-}, \vec{r}_{+}(1)}}{\partial X} \frac{\partial}{\partial \kappa_{B}} f_{d}\left(\kappa_{B}, \kappa_{B}, 0\right)}{\left[f_{d}\left(\kappa_{B}, \kappa_{B}, 0\right)\right]^{3 / 2}} .
\end{aligned}
$$

The derivative of the first order correction to the binding energy with respect to $X$ is

$$
\frac{\partial \kappa_{\vec{r}_{-}, \vec{r}_{+}(1)}}{\partial X}=\frac{\frac{2 M}{m_{1}} \frac{\partial}{\partial r_{+}} y_{d, 0}\left(\kappa_{B}, r_{+}\right)+\frac{2 M}{m_{2}} \frac{\partial}{\partial r_{-}} y_{d, 0}\left(\kappa_{B}, r_{-}\right)}{\frac{\partial}{\partial \kappa_{B}} y_{d, 0}\left(\kappa_{B}, 0\right)} \text {. }
$$

The second term in Eq. 29, due to two derivatives with respect to the spatial coordinates without any contribution from the first-order corrections $A_{\vec{r}_{-}, \vec{r}_{+}(1)}$ and $\kappa_{\vec{r}_{-}, \vec{r}_{+}(1)}$, is

$$
\begin{aligned}
T_{(B)}^{(2)}(X) & =-\frac{M}{\mu^{2} f_{d}\left(\kappa_{B}, \kappa_{B}, 0\right)} y_{d, 0}\left(\kappa_{B}, r_{+}+r_{-}\right) \\
& +\frac{r_{+}+r_{-}}{\mu f_{d}\left(\kappa_{B}, \kappa_{B}, 0\right)} y_{d,-1}\left(\kappa_{B}, r_{+}+r_{-}\right),
\end{aligned}
$$

The third term in Eq. 29, proportional to $\kappa_{\vec{r}_{-}, \vec{r}_{+}(1)}$ and $A_{\vec{r}_{-}, \vec{r}_{+}(1)}$, is

$$
\begin{aligned}
& T_{(C)}^{(2)}(X)=\left.\frac{M \kappa_{\vec{r}_{-}, \vec{r}_{+}(1)}}{2 m_{1}^{2} f_{d}(\kappa, \kappa, 0)} \frac{\partial}{\partial \kappa}\left[y_{d, 0}\left(\kappa, r_{+}\right)\right]\right|_{\kappa=\kappa_{B}}+\left.\frac{M \kappa_{\vec{r}_{-}, \vec{r}_{+}}(1)}{2 m_{2}^{2} f_{d}(\kappa, \kappa, 0)} \frac{\partial}{\partial \kappa}\left[y_{d, 0}\left(\kappa, r_{-}\right)\right]\right|_{\kappa=\kappa_{B}} \\
& +\frac{A_{\vec{r}_{-}, \vec{r}_{+}(1)}}{\left[f_{d}\left(\kappa_{B}, \kappa_{B}, 0\right)\right]^{1 / 2}}\left[\frac{M}{m_{1}^{2}} y_{d, 0}\left(\kappa_{B}, r_{+}\right)+\frac{M}{m_{2}^{2}} y_{d, 0}\left(\kappa_{B}, r_{-}\right)\right] \text {, }
\end{aligned}
$$

where $y_{d, 1}(\kappa, r), y_{d, 2}(\kappa, r)$, and $y_{d, 3}(\kappa, r)$ are the generalized Yukawa functions defined later, in Eqs. (43), (52), and (59), for $d=1,2$, and 3. For the terms in the second line of Eq.(29), we find

$$
\begin{aligned}
T_{(D 1)}^{(2)}(X) & =-\frac{\kappa_{B}}{m_{1} f_{d}\left(\kappa_{B}, \kappa_{B}, 0\right)} \frac{\partial \kappa_{\vec{r}_{-}, \vec{r}_{+}(1)}}{\partial X}\left\{\frac{r_{+}^{2}}{8} y_{d, 1}\left(\kappa_{B}, r_{+}\right)\right. \\
& \left.+\frac{r_{+}}{2} y_{d, 2}\left(\kappa_{B}, r_{+}\right)+\frac{3}{4} y_{d, 3}\left(\kappa_{B}, r_{+}\right)\right\}
\end{aligned}
$$




$$
\begin{gathered}
T_{(D 2)}^{(2)}(X)=-\frac{\kappa_{B}}{m_{2} f_{d}\left(\kappa_{B}, \kappa_{B}, 0\right)} \frac{\partial \kappa_{\vec{r}_{-}, \vec{r}_{+}(1)}}{\partial X}\left\{\frac{r_{-}^{2}}{8} y_{d, 1}\left(\kappa_{B}, r_{-}\right)\right. \\
\left.+\frac{r_{-}}{2} y_{d, 2}\left(\kappa_{B}, r_{-}\right)+\frac{3}{4} y_{d, 3}\left(\kappa_{B}, r_{-}\right)\right\} \\
T_{(D 3)}^{(2)}(X)=\frac{\kappa_{B}}{4 m_{1} f_{d}\left(\kappa_{B}, \kappa_{B}, 0\right)} \frac{\partial \kappa_{\vec{r}_{-}, \vec{r}_{+}(1)}^{\partial X}\left[r_{+} y_{d, 2}\left(\kappa_{B}, r_{+}\right)+3 y_{d, 3}\left(\kappa_{B}, r_{+}\right)\right],}{T_{(D 4)}^{(2)}(X)=\frac{\kappa_{B}}{4 m_{2} f_{d}\left(\kappa_{B}, \kappa_{B}, 0\right)} \frac{\partial \kappa_{\vec{r}_{-}, \vec{r}_{+}(1)}}{\partial X}\left[r_{-} y_{d, 2}\left(\kappa, r_{-}\right)+3 y_{d, 3}\left(\kappa, r_{-}\right)\right],} \\
T_{(E)}^{(2)}(X)=\frac{\left(\frac{\partial \kappa_{\vec{r}_{-}, \vec{r}_{+}(1)}}{\partial X}\right)^{2}}{24 M \kappa_{B}^{3} f_{d}\left(\kappa_{B}, \kappa_{B}, 0\right)}\left\{-3 \frac{\partial y_{d, 0}}{\partial \kappa_{B}}\left(\kappa_{B}, 0\right)\right. \\
\left.+3 \kappa \frac{\partial^{2} y_{d, 0}}{\partial \kappa_{B}^{2}}\left(\kappa_{B}, 0\right)-\kappa^{2} \frac{\partial^{3} y_{d, 0}}{\partial \kappa_{B}^{3}}\left(\kappa_{B}, 0\right)\right\}
\end{gathered}
$$

and the effective Hamiltonian is

$$
H_{\mathrm{eff}}^{(2)}=-\frac{1}{2 M} \frac{\partial^{2}}{\partial X^{2}}-\frac{\kappa_{B}}{\mu} \kappa_{\vec{r}_{-}, \vec{r}_{+}(2)}-\frac{1}{2 \mu} \kappa_{\vec{r}_{-}, \vec{r}_{+}(1)}^{2}+T^{(2)}(X)
$$

where $\kappa_{\vec{r}_{-}, \vec{r}_{+}(1)}, \kappa_{\vec{r}_{-}, \vec{r}_{+}(2)}$, and $T^{(2)}(X)$ are as defined above.

\section{RESULTS}

In this section we present the results of our effective field theory expansions in one, two, and three dimensions. Using the definitions given below for the generalized Yukawa function, full two Yukawa function overlap integral, and effective Hamiltonia terms from the previous section, we present the expressions for the effective Hamiltonian in one, two, and three dimensions. In all cases at zeroth order we start with the infinite volume result,

$$
H_{\mathrm{eff}}^{(0)}=-\frac{1}{2 M} \frac{\partial^{2}}{\partial X^{2}}+V_{X}^{(0)}+T_{X}^{(0)}
$$

where

$$
V_{X}^{(0)}+T_{X}^{(0)}=E_{B}
$$




\section{A. Results in One Dimension}

The generalized Yukawa function in one dimension is

$$
y_{1,0}(\kappa, r)=\frac{e^{-\kappa r}}{2 \kappa},
$$

and the full two Yukawa function overlap integral in one dimension is

$$
f_{1}(\kappa, \kappa, r)=\frac{e^{-\kappa r}}{4 \kappa^{3}}(1+\kappa r) .
$$

\section{First Order Effective Hamiltonian in One Dimension}

At first order the effective Hamiltonian for the one-dimensional case is

$$
H_{\mathrm{eff}}^{(1)}=H_{\mathrm{eff}}^{(0)}+V_{X}^{(1)}+T_{X}^{(1)},
$$

where the effective potential is

$$
V^{(1)}(X)+T^{(1)}(X)=\frac{\kappa_{B}^{2} M^{2}}{m_{1} m_{2}}\left[\frac{e^{-\kappa_{B} r_{+}(X)}}{m_{1}}+\frac{e^{-\kappa_{B} r_{-}(X)}}{m_{2}}\right]
$$

\section{Second Order Effective Hamiltonian in One Dimension}

At second order in one dimension the effective Hamiltonian is

$$
H_{\mathrm{eff}}^{(2)}=H_{\mathrm{eff}}^{(1)}+T^{(2)}(X)+V^{(2)}(X),
$$

where

$$
\begin{gathered}
V^{(2)}(X)=\frac{M \kappa_{B}^{2}}{2 m_{1} m_{2}}\left(V_{-}^{(2)}(X)+V_{+}^{(2)}(X)+V_{(A)}^{(2)}(X)\right) \\
V_{ \pm}^{(2)}(X)=e^{-2 \kappa_{B} r_{ \pm}}\left(2 \kappa_{B} r_{ \pm}-1\right) \\
V_{(A)}^{(2)}(X)=e^{-\kappa_{B}\left(r_{+}+r_{-}\right)}\left(\kappa_{B} r_{+}+\kappa_{B} r_{-}-3\right)
\end{gathered}
$$

and 


$$
\begin{aligned}
T^{(2)}(X) & =T_{(A)}^{(2)}(X)+T_{(B)}^{(2)}(X)+T_{(C)}^{(2)}(X) \\
& +T_{(D 1)}^{(2)}(X)+T_{(D 2)}^{(2)}(X)+T_{(D 3)}^{(2)}(X)+T_{(D 4)}^{(2)}(X)+T_{(E)}^{(2)}(X) .
\end{aligned}
$$

For the definitions of $T_{(A)}^{(2)}, T_{(B)}^{(2)}, T_{(C)}^{(2)}, T_{(D 1-D 4)}^{(2)}$, and $T_{(E)}^{(2)}$, see Appendix B.

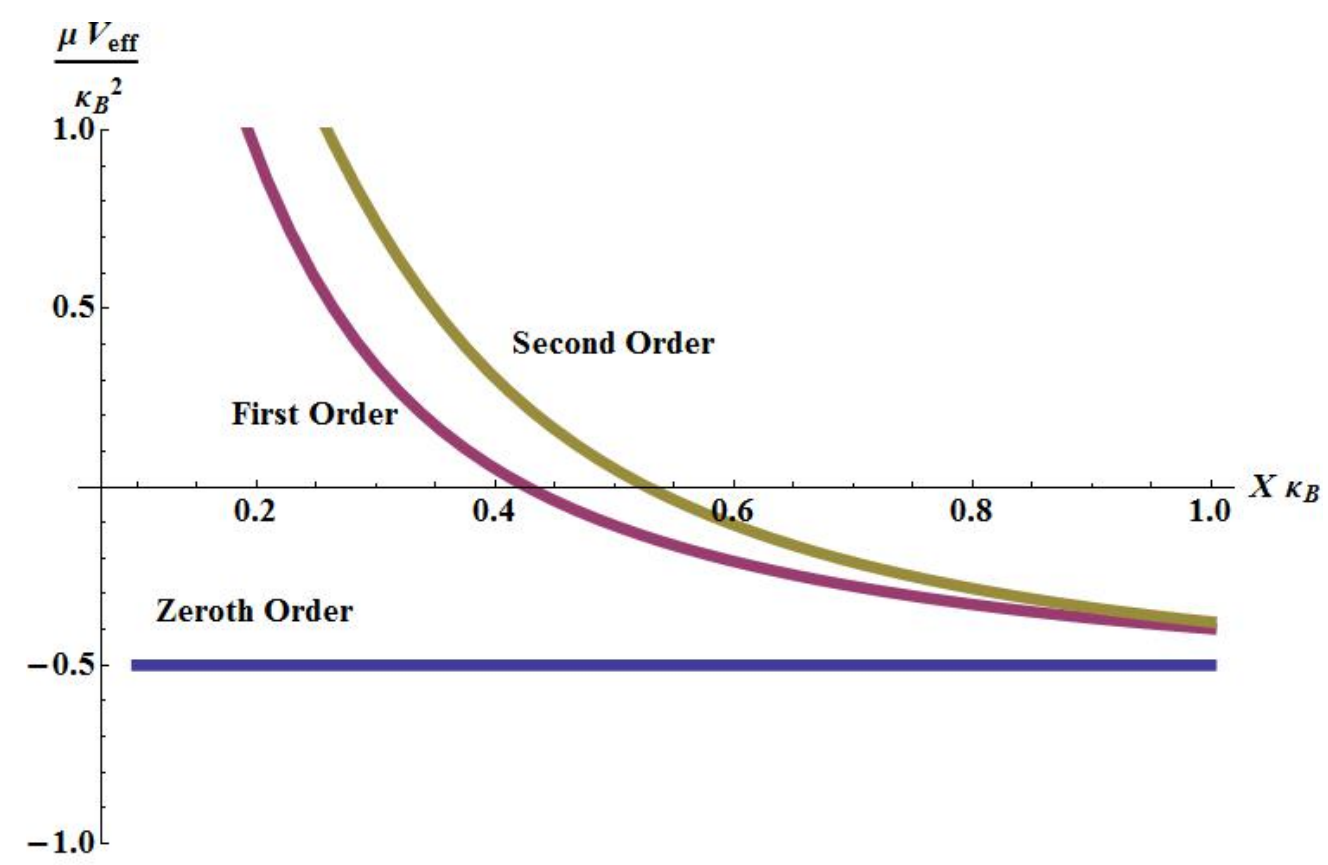

FIG. 3: Plot of the effective potential at zeroth, first, and second order in one spatial dimension for mass ratio $m_{2} / m_{1}=4$.

Fig. 3 shows a plot of the effective potential, at zeroth, first, and second order, in one spatial dimension for mass ratio $m_{2} / m_{1}=4$. The effective potential is plotted in dimensionless units of $X \kappa_{B}$ along the horizontal axis and $\frac{\mu V_{e f f}}{\kappa_{B}^{2}}$ along the vertical axis. We see that the effective potential crosses zero at $X \kappa_{B} \sim 0.5$, and the convergence for the expansion is good for $X \kappa_{B} \gtrsim 0.5$. This part of the potential gives the dominant contribution to the reflection scattering length.

\section{B. Results in Two Dimensions}

The generalized Yukawa function in two dimensions is

$$
y_{2,0}(\kappa, r)=\frac{1}{2 \pi} K_{0}(\kappa r)
$$


and the full two Yukawa function overlap integral in two dimensions is

$$
f_{2}(\kappa, \kappa, r)=\frac{r}{4 \pi \kappa} K_{1}(\kappa r)
$$

where $K_{n}$ is the modified Bessel function of the $2^{\text {nd }}$ kind.

\section{First Order Effective Hamiltonian in Two Dimensions}

In two dimensions the first order effective Hamiltonian is

$$
\begin{aligned}
H_{\mathrm{eff}}^{(1)} & =H_{\mathrm{eff}}^{(0)}+\left(\frac{1}{\mu}+\frac{M}{m_{1}^{2}}\right) \kappa_{B}^{2} K_{0}\left[\kappa_{B} r_{+}(X)\right] \\
& +\left(\frac{1}{\mu}+\frac{M}{m_{2}^{2}}\right) \kappa_{B}^{2} K_{0}\left[\kappa_{B} r_{-}(X)\right],
\end{aligned}
$$

where the adiabatic potential is

$$
V^{(1)}(X)=\frac{\kappa_{B}^{2}}{\mu}\left[K_{0}\left(\kappa_{B} r_{+}\right)+K_{0}\left(\kappa_{B} r_{-}\right)\right]
$$

and the diagonal adiabatic correction is

$$
T^{(1)}(X)=\frac{\kappa_{B}^{2} M}{m_{1}^{2}} K_{0}\left(\kappa_{B} r_{+}\right)+\frac{\kappa_{B}^{2} M}{m_{2}^{2}} K_{0}\left(\kappa_{B} r_{-}\right) .
$$

\section{Second Order Effective Hamiltonian in Two Dimensions}

At second order in two dimensions we find the adiabatic potential

$$
V^{(2)}(X)=-\frac{\kappa_{B}}{\mu} \kappa_{\vec{r}_{-}, \vec{r}_{+}(2)}-\frac{1}{2 \mu} \kappa_{\vec{r}_{-}, \vec{r}_{+}(1)}^{2} .
$$

The diagonal adiabatic correction is

$$
\begin{aligned}
T^{(2)}(X) & =T_{(A)}^{(2)}(X)+T_{(B)}^{(2)}(X)+T_{(C)}^{(2)}(X) \\
& +T_{(D 1)}^{(2)}(X)+T_{(D 2)}^{(2)}(X)+T_{(D 3)}^{(2)}(X)+T_{(D 4)}^{(2)}(X)+T_{(E)}^{(2)}(X) .
\end{aligned}
$$

For the definitions of $\kappa_{\vec{r}_{-}, \vec{r}_{+}(1)}, \kappa_{\vec{r}_{-}, \vec{r}_{+}(2)}, T_{(A)}^{(2)}, T_{(B)}^{(2)}, T_{(C)}^{(2)}, T_{(D 1-D 4)}^{(2)}$, and $T_{(E)}^{(2)}$, see Appendix C. 


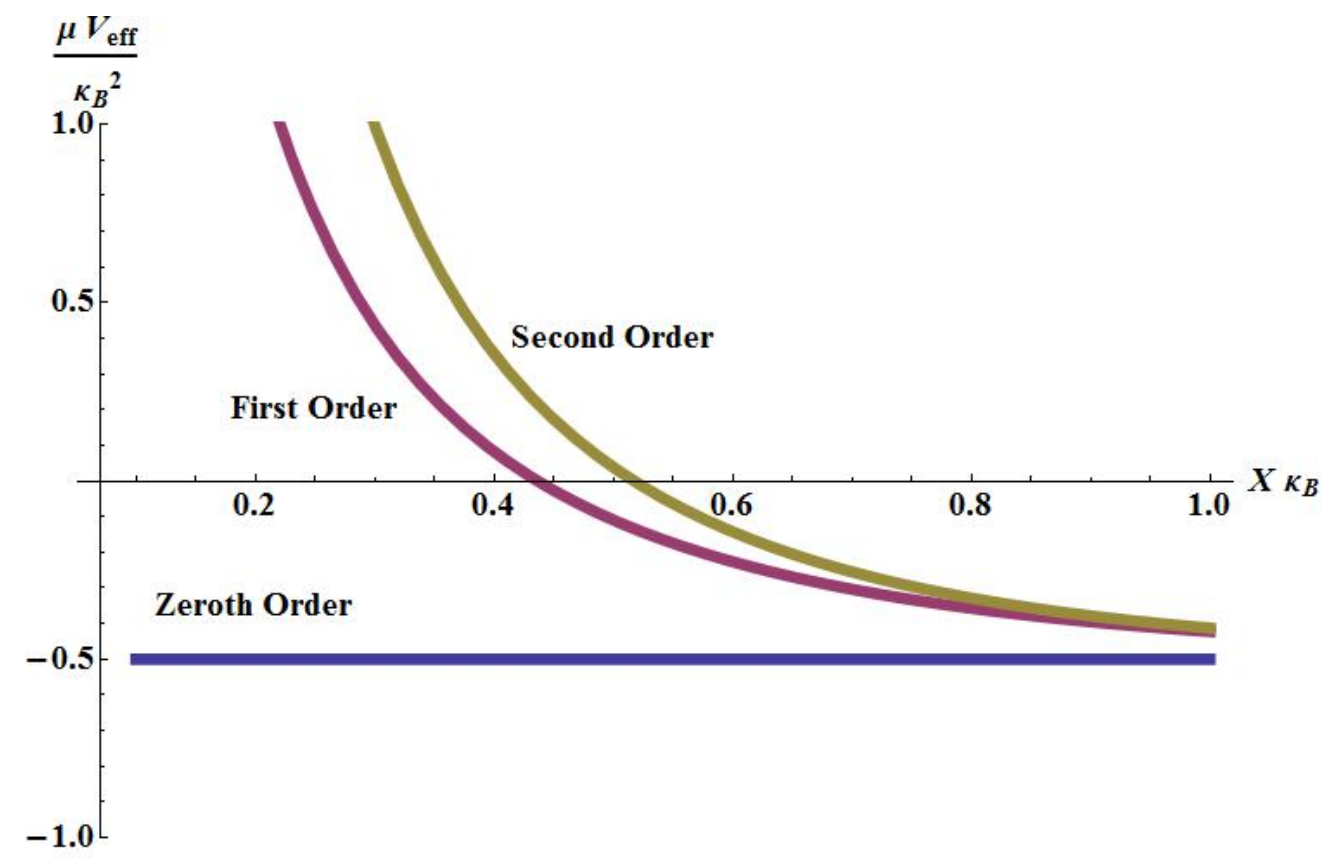

FIG. 4: Plot of the effective potential at zeroth, first, and second order in two spatial dimensions for mass ratio $m_{2} / m_{1}=4$.

Fig. 4 shows a plot of the effective potential in two spatial dimensions for mass ratio $m_{2} / m_{1}=4$. The effective potential is plotted in dimensionless units of $X \kappa_{B}$ along the horizontal axis and $\frac{\mu V_{e f f}}{\kappa_{B}^{2}}$ along the vertical axis. Again the effective potential crosses zero at $X \kappa_{B} \sim 0.5$, and the convergence of the expansion is good for larger values of $X \kappa_{B}$.

\section{Results in Three Dimensions}

The generalized Yukawa function in three dimensions is

$$
y_{3,0}(\kappa, r)=\frac{e^{-\kappa r}}{4 \pi r}
$$

and the full two Yukawa function overlap integral in three dimensions is

$$
f_{3}(\kappa, \kappa, r)=\frac{e^{-\kappa r}}{8 \pi \kappa} .
$$

\section{First Order Effective Hamiltonian in Three Dimensions}

In three dimensions at first order the effective Hamiltonian is 


$$
H_{\mathrm{eff}}^{(1)}=H_{\mathrm{eff}}^{(0)}+T^{(1)}(X)+V^{(1)}(X),
$$

where the effective potential is

$$
T^{(1)}(X)+V^{(1)}(X)=\frac{\kappa_{B} M}{2 m_{1} m_{2} X}\left[e^{-\kappa_{B} r_{+}(X)}+e^{-\kappa_{B} r_{-}(X)}\right] .
$$

\section{Second Order Effective Hamiltonian in Three Dimensions}

At second order in three dimensions we find that the adiabatic potential is

$$
V^{(2)}(X)=-\frac{\kappa_{B}}{\mu} \kappa_{\vec{r}_{-}, \vec{r}_{+}(2)}-\frac{1}{2 \mu} \kappa_{\vec{r}_{-}, \vec{r}_{+}(1)}^{2} .
$$

For the diagonal adiabatic correction, we find

$$
\begin{aligned}
T^{(2)}(X) & =T_{(A)}^{(2)}(X)+T_{(B)}^{(2)}(X)+T_{(C)}^{(2)}(X) \\
& +T_{(D 1)}^{(2)}(X)+T_{(D 2)}^{(2)}(X)+T_{(D 3)}^{(2)}(X)+T_{(D 4)}^{(2)}(X)+T_{(E)}^{(2)}(X),
\end{aligned}
$$

For the definitions of $\kappa_{\vec{r}_{-}, \vec{r}_{+}(1)}, \kappa_{\vec{r}_{-}, \vec{r}_{+}(2)}, T_{(A)}^{(2)}, T_{(B)}^{(2)}, T_{(C)}^{(2)}, T_{(D 1-D 4)}^{(2)}$, and $T_{(E)}^{(2)}$, see Appendix D.

Fig. 5 shows a plot of the effective potential in three spatial dimensions for mass ratio $m_{2} / m_{1}=4$. As in the one and two dimensional cases, the effective potential is plotted in dimensionless units of $X \kappa_{B}$ along the horizontal axis and $\frac{\mu V_{e f f}}{\kappa_{B}^{2}}$ along the vertical axis. The effective potential crosses the horizontal axis at $X \kappa_{B} \sim 0.4$, and the convergence of the expansion is good for $X \kappa_{B} \gtrsim 0.4$.

\section{SCATTERING LENGTH AND REFLECTION RADIUS}

From these effective potentials it is straightforward to compute the reflection scattering length up to second order. This process can be carried forward to any order. The net result is an expansion with an expansion parameter of size $e^{-\kappa_{B} r_{ \pm}\left(a_{R}\right)} \leq e^{-2 \kappa_{B} a_{R}}$. The larger $\kappa_{B} a_{R}$, the faster the convergence of the expansion. First- and second-order results for the one-dimensional system are shown in Fig. 6. Results for the two-dimensional system are shown in Fig. 7, and results for the three-dimensional system are shown in Fig. 8, In each 


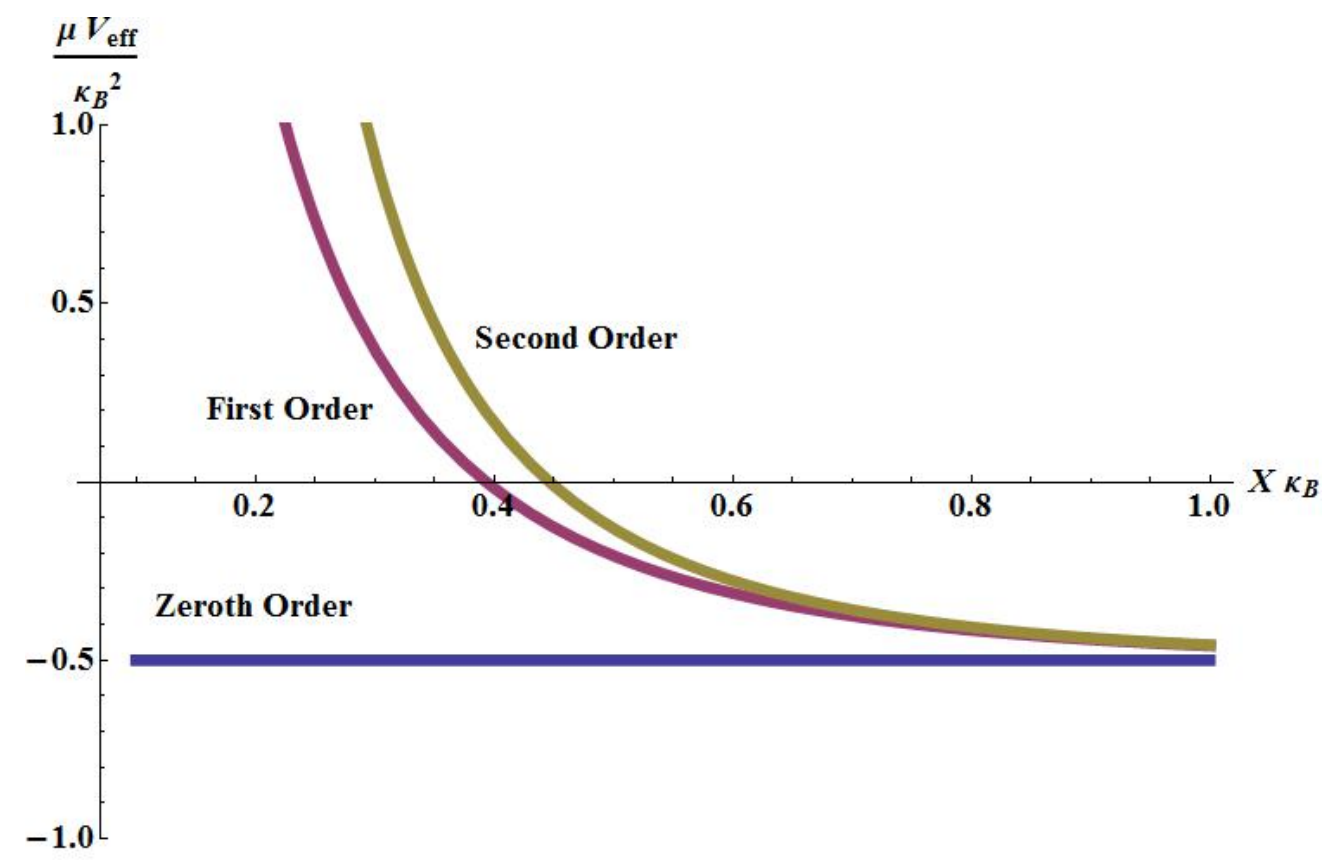

FIG. 5: Plot of the effective potential at zeroth, first, and second order in three spatial dimensions for mass ratio $m_{2} / m_{1}=4$.

case the agreement with lattice results is consistent with third-order corrections of size $\leq$ $e^{-6 \kappa_{B} a_{R}}$.

\section{A. Numerical Results}

We have calculated the dimer-wall reflection phase shift using Hamiltonian lattice methods in one, two, and three dimensions. We implement the two-particle interaction as an attractive point-like interaction. The lattice Hamiltonian in $d$ dimensions is given by

$$
\begin{aligned}
H & =\frac{d}{m} \sum_{i=1,2} \sum_{\vec{n}} a_{i}^{\dagger}(\vec{n}) a_{i}(\vec{n}) \\
& -\frac{1}{2 m} \sum_{i=1,2} \sum_{\vec{n}} \sum_{l=1}^{d}\left[a_{i}^{\dagger}(\vec{n}) a_{i}(\vec{n}+\hat{l})+a_{i}^{\dagger}(\vec{n}) a_{i}(\vec{n}-\hat{l})\right] \\
& +c \sum_{\vec{n}} a_{1}^{\dagger}(\vec{n}) a_{1}(\vec{n}) a_{2}^{\dagger}(\vec{n}) a_{2}(\vec{n})
\end{aligned}
$$

where $a_{i}^{\dagger}$ and $a_{i}$ are the creation and annihilation operators for particle $i, \vec{n}$ labels the lattice site, and $\hat{l}$ is the unit vector in the $l^{\text {th }}$ direction. We take parameters and operators to be 
dimensionless by multiplying physical values by the appropriate power of the lattice spacing.

Two parallel hard-wall boundaries are spaced a distance $L_{z}$ apart. We impose periodic boundary conditions in the perpendicular directions with length $L$, and consider dimer states where the momentum is perpendicular to the wall. We calculate the confinement energy as a function of $L_{z}$ using sparse-matrix eigenvector methods and from this calculation determine the reflection phase shift using Eq. (2). For various mass ratios of the two particles, we repeat our calculations using successively smaller lattice spacings. This allows us to extrapolate the reflection radius and dimer kinetic energy to the continuum limit and determine universal results in the shallow-binding limit. For the two- and three-dimensional cases we also perform infinite volume extrapolations in the dimension perpendicular to the wall.

The coefficient of the delta function interaction in Eq. (65) is $c$. In order to take the continuum limit in one dimension, we fix $L_{z}|c|$ and extrapolate both $R \kappa_{B}$ and $E_{K} /\left|E_{B}\right|$ for three different values of $L$. The values of $L_{z}|c|$ we consider are $L_{z}|c|=8,10,20$. For $L_{z}|c|=8$, we calculate the ground state values for $R \kappa_{B}$ and $E_{K} /\left|E_{B}\right|$ for $L_{z}=80,160,240$. For $L_{z}|c|=10$, we consider the ground state and first two excited states for $L_{z}=100,200,300$. For $L_{z}|c|=20$, we consider the ground state for $L_{z}=200,300,400$.

In two and three dimensions, we first take the infinite volume limit in the perpendicular directions by extrapolating $L \rightarrow \infty$. We then extrapolate to the continuum limit using several values for $L_{z}$ while keeping $\kappa_{B} L_{z}$ fixed, where $\kappa_{B}$ is the dimer binding momentum. In two dimensions we consider the ground state and first excited state for $L_{z} \kappa_{B}=6.8,10.2$. For $L_{z} \kappa_{B}=6.8$ we perform calculations using $L_{z}=40,60,80,100$, and for $L_{z} \kappa_{B}=10.2$ we consider $L_{z}=60,90,120,150$.

In three dimensions we consider the ground state and first excited state for $L_{z} \kappa_{B}=$ 4.9, 6.0. For $L_{z} \kappa_{B}=4.9$ we perform calculations using $L_{z}=20,30,40$, and for $L_{z} \kappa_{B}=6.0$ we consider $L_{z}=24,36,48$. For a summary of the continuum extrapolations performed, including the lattice volumes used, see Appendix E.

\section{Numerical Results in One Dimension}

Fig. 6 shows the one-dimensional results in the shallow-binding limit, plotted as the reflection radius versus dimer kinetic energy, $E_{K}$, in dimensionless units, $\kappa_{B} R\left(E_{K}\right)$ versus $E_{K} /\left|E_{B}\right|$. The results are plotted at several different mass ratios, $m_{2} / m_{1}$. As expected 
the reflection radius decreases monotonically with increasing energy. One interesting feature is the dependence on the mass ratio. For larger $m_{2} / m_{1}$ the reflection radius is larger but decreases quickly with increasing energy. This indicates that a bound state with constituent particle masses that are very different will behave like a large but soft deformable ball upon contact with the wall boundary.

When $m_{2} / m_{1}=1$ in one dimension the problem is integrable and exactly solvable via the Bethe Ansatz. From the Bethe Ansatz we get

$$
\begin{gathered}
p \cot \delta(p)=-2 \kappa_{B}, \\
\kappa_{B} R\left(E_{K}\right)=\frac{1}{2} \sqrt{\frac{\left|E_{B}\right|}{E_{K}}} \tan ^{-1} \sqrt{\frac{E_{K}}{\left|E_{B}\right|}} .
\end{gathered}
$$

As shown in Fig. 6, the lattice results agree with the solution given by the Bethe Ansatz.

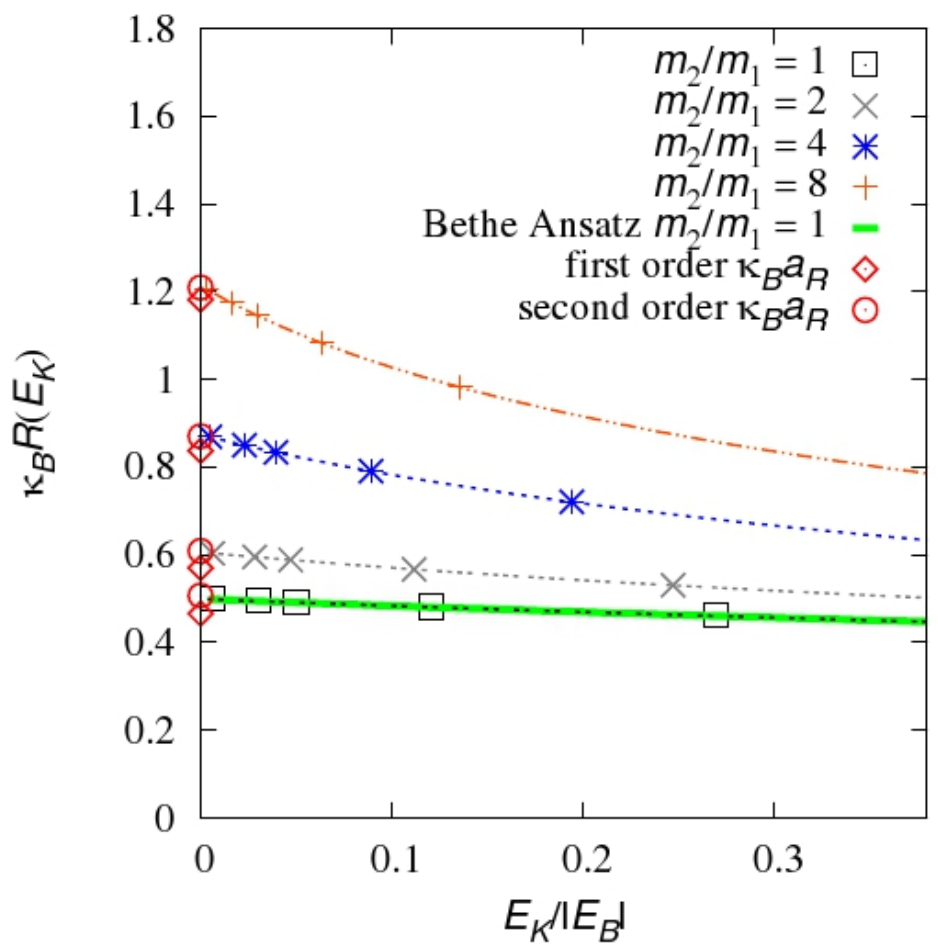

FIG. 6: One-dimensional lattice results for the reflection radius versus dimer kinetic energy in the shallow-binding limit. Also shown are Bethe Ansatz results for $m_{2} / m_{1}=1$ and first- and second-order results for the expansion of $\kappa_{B} a_{R}$ described in the text.

Fig. 6 also shows the first- and second-order analytic results for the expansion of $\kappa_{B} a_{R}$ 


\begin{tabular}{|c|c|c|c|}
$m_{2} / m_{1}$ & $\kappa_{B} a_{R}$ & $\kappa_{B} r_{R}$ & $\kappa_{B}^{3} P_{R}$ \\
\hline \hline 1 & $0.4999(2)$ & $0.005(7)$ & $0.002(3)$ \\
2 & $0.6065(2)$ & $-0.074(2)$ & $-0.006(2)$ \\
4 & $0.8747(2)$ & $0.115(2)$ & $0.006(2)$ \\
8 & $1.2149(2)$ & $0.460(2)$ & $0.008(2)$
\end{tabular}

TABLE I: Coefficients of the effective range expansion for one-dimensional dimer-wall scattering.

derived from our general effective theory.

Table II presents the coefficients of the effective range expansion for one-dimensional dimer-wall reflection. The error estimates are from the least-squares fitting used in the lattice extrapolation and the effective range expansion. From Eq. (66), we see that the Bethe Ansatz gives $\kappa_{B} a_{R}=1 / 2$ for $m_{2} / m_{1}=1$, with all other coefficients equal to zero. This completely agrees with the lattice results. These results are universal and could be verified using other theoretical methods or experiments such as cold atomic dimers confined to a one-dimensional optical lattice with sharp boundaries.

\section{Numerical Results in Two Dimensions}

Fig. 7 shows the reflection radius versus dimer kinetic energy for the two-dimensional system in the shallow-binding limit. Again the results are presented in dimensionless combinations, $\kappa_{B} R\left(E_{K}\right)$ versus $E_{K} /\left|E_{B}\right|$. The reflection radius is somewhat smaller than in the one-dimensional case. This is reasonable considering the difference between compression of a one-dimensional object versus compression of a two-dimensional object along just one dimension. In the first case, we expect the object to resist compression more since it does not have another direction to expand along as it is compressed. As in the one-dimensional case, we see the same dependence on the mass ratio. At large $m_{2} / m_{1}$ the reflection radius is large at small energies while becoming substantially smaller with increasing energy. Fig. 7 also shows the first- and second-order results for the asymptotic expansion of $\kappa_{B} a_{R}$ derived from the general theory for shallow two-body bound state reflection.

The coefficients of the effective range expansion for the two-dimensional system are given in Table 凹. 


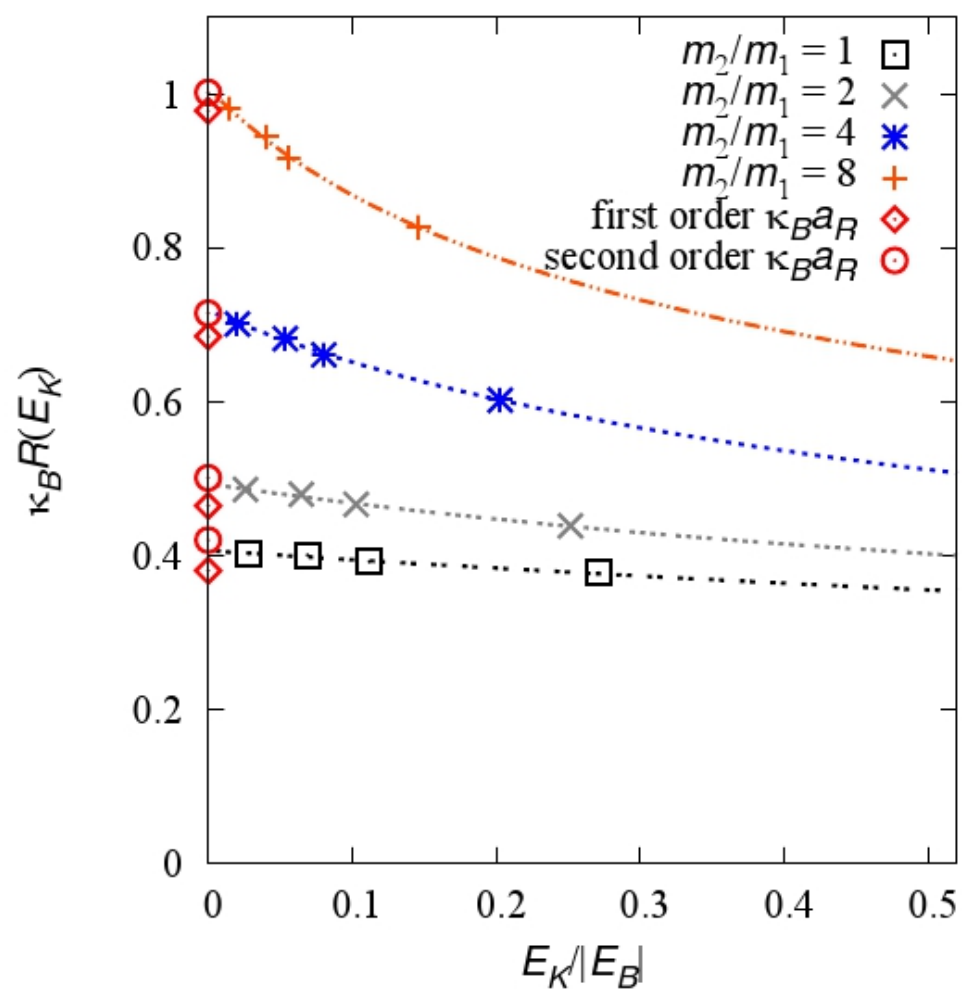

FIG. 7: Two-dimensional lattice results for the reflection radius vs dimer kinetic energy in the shallow binding-energy limit. Also shown are first- and second- order results for the expansion of $\kappa_{B} a_{R}$ described later in the text.

\begin{tabular}{|c|c|c|c|}
$m_{2} / m_{1}$ & $\kappa_{B} a_{R}$ & $\kappa_{B} r_{R}$ & $\kappa_{B}^{3} P_{R}$ \\
\hline \hline 1 & $0.407(5)$ & $-0.10(28)$ & $0.00(11)$ \\
2 & $0.494(5)$ & $-0.18(19)$ & $-0.01(7)$ \\
4 & $0.718(6)$ & $-0.01(10)$ & $-0.00(3)$ \\
8 & $1.009(9)$ & $0.29(6)$ & $-0.001(17)$
\end{tabular}

TABLE II: Coefficients of the effective range expansion for two-dimensional dimer-wall scattering

3. Numerical Results in Three Dimensions

Fig. 8 shows the reflection radius versus dimer kinetic energy for the three-dimensional system in the shallow-binding limit. Again the results are presented in dimensionless combi- 


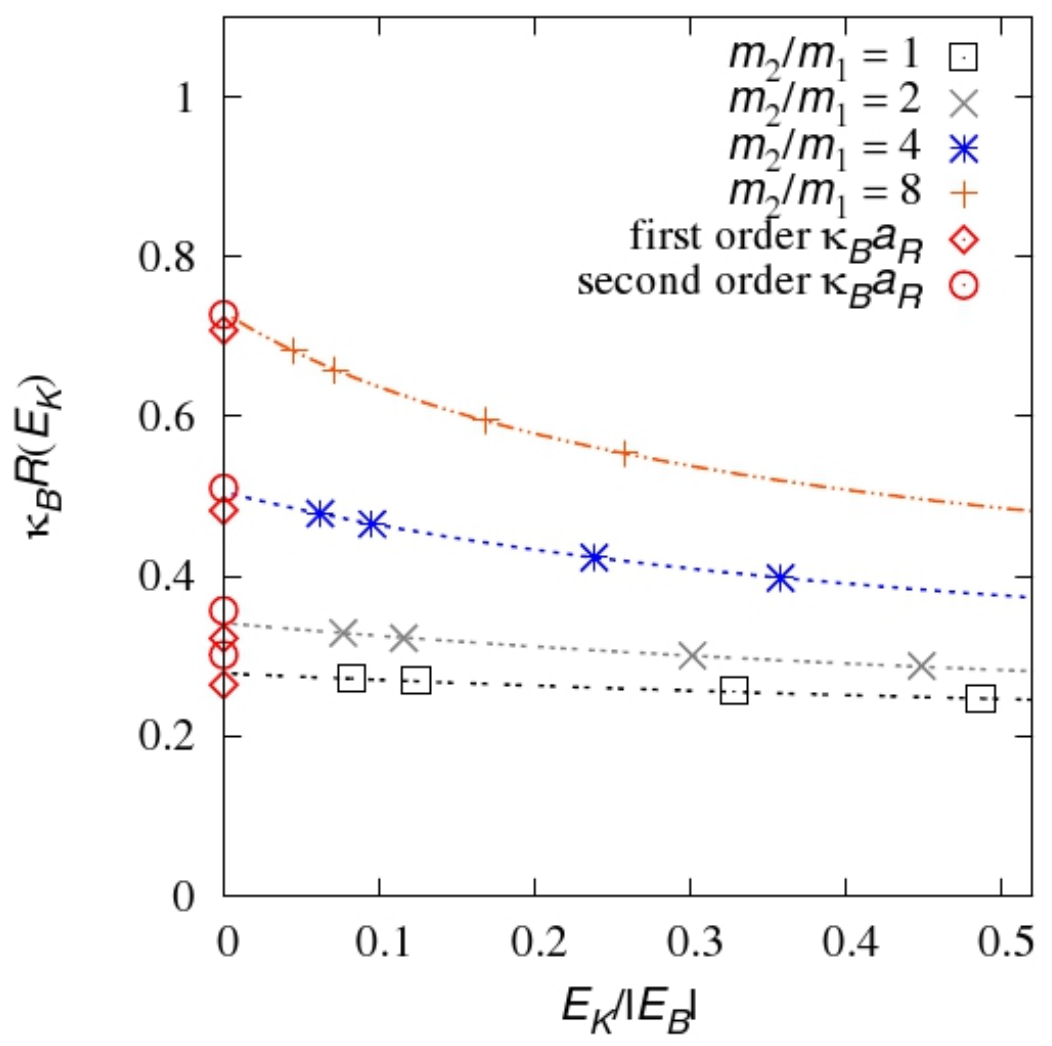

FIG. 8: Three-dimensional lattice results for the reflection radius versus dimer kinetic energy in the shallow-binding limit. Also shown are first- and second-order results for $\kappa_{B} a_{R}$ described in the text.

nations, $\kappa_{B} R\left(E_{K}\right)$ versus $E_{K} /\left|E_{B}\right|$. The results are plotted at several different mass ratios, $m_{2} / m_{1}$. Again the reflection radius is smaller than in the two-dimensional case and nearly a factor of two smaller than in the one-dimensional case. Just as in the two-dimensional case, this conforms to our expectation that the three-dimensional object will resist compression even less than the two dimensional object, since it has two unconstrained dimensions it can expand along rather than just one. As in the one- and two-dimensional cases, we once again find a direct relationship between mass ratio and the derivative of the reflection radius with respect to the dimer kinetic energy. Fig. 8 also shows the first- and second-order results for the asymptotic expansion of $\kappa_{B} a_{R}$ derived from the general theory for shallow two-body bound state reflection. The coefficients of the effective range expansion for the three-dimensional system are given in Table III.

We now address what happens in the limit $m_{2} / m_{1} \rightarrow \infty$. Consider the limit $m_{2} \rightarrow \infty$ 


\begin{tabular}{|c|c|c|c|}
$m_{2} / m_{1}$ & $\kappa_{B} a_{R}$ & $\kappa_{B} r_{R}$ & $\kappa_{B}^{3} P_{R}$ \\
\hline \hline 1 & $0.279(8)$ & $-0.38(9)$ & $-0.02(2)$ \\
2 & $0.342(8)$ & $-0.44(6)$ & $-0.02(2)$ \\
4 & $0.506(8)$ & $-0.27(4)$ & $-0.02(1)$ \\
8 & $0.731(8)$ & $0.03(2)$ & $-0.005(5)$
\end{tabular}

TABLE III: Coefficients of the effective range expansion for three-dimensional dimer-wall scattering.

\begin{tabular}{|c|c|c|}
$L$ & $p(L)$ & $R[p(L)]$ \\
\hline \hline $11.8 \mathrm{fm}$ & $81(9) \mathrm{MeV}$ & $2.1(4) \mathrm{fm}$ \\
$9.9 \mathrm{fm}$ & $97(10) \mathrm{MeV}$ & $1.6(3) \mathrm{fm}$ \\
$7.9 \mathrm{fm}$ & $118(10) \mathrm{MeV}$ & $1.3(2) \mathrm{fm}$
\end{tabular}

TABLE IV: Momenta and reflection radii for an alpha particle confined to a cube of length $L$.

with $m_{1}$ held fixed. In this limit the effective potential converges to a non-vanishing finitevalued function, while the mass of the dimer grows with $m_{2}$. One can check this explicitly for the expressions in Eq. (46) and Eq. (62). Given the exponential tail of the effective potential, the reflection radius near threshold has a logarithmic dependence on $m_{2} / m_{1}$. We see this behavior in each of the plots in Fig. 6, 7, and 8 .

\section{Alpha Particle}

Using lattice effective field theory, we have calculated the energy of an alpha particle in a cubic box at leading order in chiral effective field theory at lattice spacing $a=1.97 \mathrm{fm}$ for cubic boxes of lengths $L=11.8 \mathrm{fm}, 9.9 \mathrm{fm}$, and $7.9 \mathrm{fm}$. We use the same lattice action, algorithms, and codes as in Ref. [16]. A review of lattice effective field theory can be found in Ref. [24]. The vanishing Dirichlet boundaries are implemented as an essentially infinite potential energy at the wall boundaries. We note that the ultraviolet divergences are independent of long-distance boundary conditions, and so no new renormalization counterterms are needed. In Fig. 9 we plot the energy expectation value of the alpha particle as a function of the Euclidean time projection. The exponential curves shown are best fits to a functional 


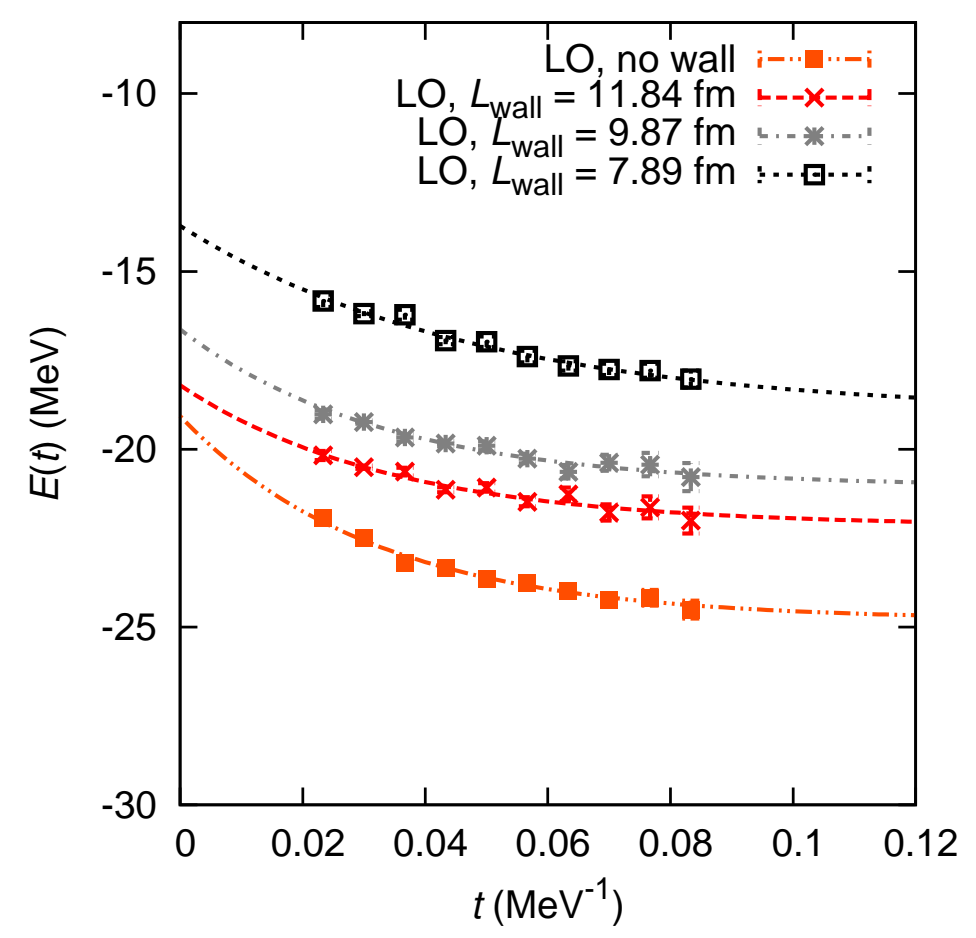

FIG. 9: Energy of an alpha particle confined to cubic box of lengths 11.8, 9.9, and $7.9 \mathrm{fm}$.

form

$$
E(t)=E_{0}+c e^{-\Delta E t}
$$

These capture the asymptotic behavior at large $t$.

Using the asymptotic form in Eq. (68) we extract the ground state energy $E_{0}$ for the given boundary conditions. Using Eqs. (2) and (3), we can calculate $P(L)$ and $R[P(L)]$. The results are shown in Table IV. The error bars in Table IV are one standard deviation estimates which include both Monte Carlo statistical errors and uncertainties due to extrapolation at large Euclidean time. At leading order we find the root-mean-square (RMS) matter radius for point-like nucleons to be $1.53(4) \mathrm{fm}$. Comparing the reflection radii in Table IV to the RMS matter radius of the alpha particle, we find that at low momenta the reflection radius of the alpha particle is larger than the RMS matter radius. However for larger momenta we see a substantial decrease in the alpha particle reflection radius. This suggests that the alpha particle is quite compressible under confinement pressure. This appears consistent with the observation that alpha clusters are compressed in size when confined within nuclei. Much more numerical work is planned to study alpha particles and other nuclei under confinement 
pressure.

\section{SUMMARY AND FUTURE WORK}

In this paper we derived the phase shift due to the scattering of a two-particle bound state on a hard wall in the adiabatic limit and up to second order in an expansion of the effective Hamiltonian. We have presented the effective Hamiltonian as an expansion in the parameters $e^{-\kappa_{B} r_{+}}$and $e^{-\kappa_{B} r_{-}}$. We plotted the effective potential in one, two, and three dimensions, and discussed the convergence of the expansion. For the equal mass case in one dimension we have presented an exact analytic solution using the Bethe Ansatz method. Additionaly, we presented numerical and effective field theory calculations of the reflection radius in one, two, and three spatial dimensions. We then discussed the consistency among the analytic, numeric, and effective field theory calculations. We saw that, as expected, the bound state becomes more readily compressible as the number of spatial dimensions increases. Furthermore, we noted an interesting mass ratio dependence of the reflection radius, noting that for larger mass ratios the reflection radius is larger but decreases faster as the kinetic energy of the bound state increases. Using lattice effective field theory we calculated the alpha particle energy in a cubic box for $L=11.8 \mathrm{fm}, 9.9 \mathrm{fm}$, and $7.9 \mathrm{fm}$. We then calculated the alpha particle reflection radius and in our analysis of the alpha particle compressibility we found that the alpha particle actually appears to compress rather easily.

We have discussed many aspects of the elastic scattering of quantum bound states from a hard surface, paying particular attention to universal behavior which is common to many different systems. There appear to be many applications, ranging from experimental predictions for quantum dots and wells to numerical calculations of nuclear structure and elastic deformation. The theoretical techniques employed in this analysis may prove useful in describing the effective field theory of other inhomogeneous systems. One immediate extension of our three-dimensional analysis is to calculate the deuteron reflection phase shift from $a b$ initio lattice chiral effective field theory to verify the universal effective range expansion coefficients in Table III, as well as to measure the spin dependence of the reflection phase shift resulting from the $D$-wave component of the deuteron wavefunction. Some other interesting extensions include investigating the inelastic threshold, and varying the boundary conditions used. 
Another immediate extension is to verify the logarithmic $m_{2} / m_{1}$ threshold dependence in various physical systems. We expect this effect to be prominent for halo nuclei with a heavy core and one satellite nucleon as well as in quantum dots and wells for semiconductors with a large ratio between hole and electron effective masses. It could also be reproduced experimentally with heterogeneous cold atomic dimers consisting of one heavy and one light alkali atom.

Financial support from the U.S. Department of Energy and NC State GAANN Fellowship is acknowledged. Computational resources provided by the Jülich Supercomputing Centre at Forschungszentrum Jülich.

[1] T. Takagahara and K. Takeda, Phys. Rev. B 46, 15578 (1992).

[2] J. Y. Marzin, J. M. Gérard, A. Izraël, D. Barrier, and G. Bastard, Phys. Rev. Lett. 73, 716 (1994).

[3] S. Pfalz, R. Winkler, T. Nowitzki, D. Reuter, A. D. Wieck, D. Hägele, and M. Oestreich, Phys. Rev. B 71, 165305 (2005).

[4] L. He, G. Bester, and A. Zunger, Phys. Rev. Lett. 94, 016801 (2005).

[5] S. Montangero, D. Frustaglia, T. Calarco, and R. Razio, Europhys. Lett. 88, 30006 (2009).

[6] M. Lüscher, Commun. Math. Phys. 104, 177 (1986).

[7] M. Lüscher, Commun. Math. Phys. 105, 153 (1986).

[8] S. R. Beane, P. F. Bedaque, K. Orginos, and M. J. Savage (NPLQCD), Phys. Rev. D73, 054503 (2006), hep-lat/0506013.

[9] S. R. Beane, P. F. Bedaque, K. Orginos, and M. J. Savage, Phys. Rev. Lett. 97, 012001 (2006), hep-lat/0602010.

[10] V. Bernard, M. Lage, U.-G. Meißner, and A. Rusetsky, JHEP 08, 024 (2008), arXiv:0806.4495 [hep-lat].

[11] E. Epelbaum, H. Krebs, D. Lee, and U.-G. Meißner, Phys. Rev. Lett. 104, 142501 (2010), arXiv:0912.4195 [nucl-th].

[12] E. Epelbaum, H. Krebs, D. Lee, and U.-G. Meißner, Eur. Phys. J. A45, 335 (2010), arXiv:1003.5697 [nucl-th].

[13] A. Tohsaki, H. Horiuchi, P. Schuck, and G. Ropke, Phys. Rev. Lett. 87, 192501 (2001), nucl- 
th/0110014.

[14] M. Chernykh, H. Feldmeier, T. Neff, P. von Neumann-Cosel, and A. Richter, Phys. Rev. Lett. 98, 032501 (2007).

[15] Y. Suzuki et al., Phys. Lett. B659, 160 (2008), nucl-th/0703001.

[16] E. Epelbaum, H. Krebs, D. Lee, and U.-G. Meißner, Phys. Rev. Lett. 106, 192501 (2011), arXiv:1101.2547 [nucl-th].

[17] D. Lee and M. Pine, Eur. Phys. J. A47, 41 (2011), 1008.5187.

[18] S. König, D. Lee, and H.-W. Hammer, Annals Phys. 327, 1450 (2012), 1109.4577.

[19] S. König, D. Lee, and H.-W. Hammer, Phys.Rev.Lett. 107, 112001 (2011), 1103.4468.

[20] S. Tan (2012), 1201.6014v1.

[21] J. Macek, J. Phys. B 1, 831 (1968).

[22] C. D. Lin, Phys. Rev. 257, 1 (1995).

[23] B. D. Esry, C. D. Lin, and C. H. Greene, Phys. Rev. A 54, 394 (1996).

[24] D. Lee, Prog. Part. Nucl. Phys. 63, 117 (2009), arXiv:0804.3501 [nucl-th].

\section{Appendix A: Derivation of the Diagonal Adiabatic Correction}

We have already defined the diagonal adiabatic correction as

$$
T(X)=-\frac{1}{2 M}\left\langle\psi_{X}\left|\frac{\partial^{2}}{\partial X^{2}}\right| \psi_{X}\right\rangle
$$

where

$$
\psi_{X}(\vec{r})=A_{\vec{r}_{-}, \vec{r}_{+}} \phi_{\vec{r}_{-}, \vec{r}_{+}}(\vec{r}) .
$$

The normalization condition gives

$$
\begin{aligned}
A_{\vec{r}_{-}, \vec{r}_{+}} & =\left[f_{d}\left(\kappa_{\vec{r}_{-}, \vec{r}_{+}}, \kappa_{\vec{r}_{-}, \vec{r}_{+}}, 0\right)-f_{d}\left(\kappa_{\vec{r}_{-}, \vec{r}_{+}}, \kappa_{\vec{r}_{-}, \vec{r}_{+}}, r_{-}\right)-f_{d}\left(\kappa_{\vec{r}_{-}, \vec{r}_{+}}, \kappa_{\vec{r}_{-}, \vec{r}_{+}}, r_{+}\right)\right. \\
& \left.+2 f_{d}\left(\kappa_{\vec{r}_{-}, \vec{r}_{+}}, \kappa_{\vec{r}_{-}, \vec{r}_{+}}, r_{+}+r_{-}\right)+\cdots\right]^{-1 / 2},
\end{aligned}
$$

where 


$$
\begin{aligned}
\phi_{\vec{r}_{-}, \vec{r}_{+}}(\vec{r}) & =y_{d, 0}\left(\kappa_{\vec{r}_{-}, \vec{r}_{+}},|\vec{r}|\right)-y_{d, 0}\left(\kappa_{\vec{r}_{-}, \vec{r}_{+}},\left|\vec{r}-\vec{r}_{-}\right|\right)-y_{d, 0}\left(\kappa_{\vec{r}_{-}, \vec{r}_{+}},\left|\vec{r}-\vec{r}_{+}\right|\right) \\
& +y_{d, 0}\left(\kappa_{\vec{r}_{-}, \vec{r}_{+}},\left|\vec{r}-\vec{r}_{+}+\vec{r}_{-}\right|\right)+y_{d, 0}\left(\kappa_{\vec{r}_{-}, \vec{r}_{+}}, \vec{r}+\vec{r}_{+}-\vec{r}_{-}\right)+\cdots
\end{aligned}
$$

and $A_{\vec{r}_{-}, \vec{r}_{+}}$is the function that normalizes the relative coordinate wavefunction, $\phi_{\vec{r}_{-}, \vec{r}_{+}}(\vec{r})$. The $d$-dimensional Yukawa function is

$$
y_{d, 0}(\kappa, r)=\int \frac{d^{d} \vec{p}}{(2 \pi)^{d}} \frac{e^{-i \vec{p} \cdot \vec{r}}}{p^{2}+\kappa^{2}}
$$

and the overlap integral is

$$
f_{d}\left(\kappa^{I}, \kappa^{I I}, r\right)=\int d^{d} \vec{r}^{\prime} y_{d, 0}\left(\kappa^{I},\left|\vec{r}^{\prime}\right|\right) y_{d, 0}\left(\kappa^{I I},\left|\vec{r}^{\prime}-\vec{r}\right|\right),
$$

for two Yukawa functions whose centers are separated by a spatial distance $r$.

We think of $\phi_{\vec{r}_{-}, \vec{r}_{+}}(\vec{r})$ as a Yukawa function centered at the origin, plus images from a single mirror reflection, plus images from two mirror reflections, and so on. We expand $A_{\vec{r}_{-}, \vec{r}_{+}}$by including more reflections at each increasing order. This procedure gives

$$
A_{\vec{r}_{-}, \vec{r}_{+}(0)}=\frac{1}{\left[f_{d}\left(\kappa_{B}, \kappa_{B}, 0\right)\right]^{1 / 2}},
$$

and

$$
\begin{aligned}
A_{\vec{r}_{-}, \vec{r}_{+}(1)} & =\frac{1}{2} \frac{f_{d}\left(\kappa_{B}, \kappa_{B}, r_{-}\right)+f_{d}\left(\kappa_{B}, \kappa_{B}, r_{+}\right)}{\left[f_{d}\left(\kappa_{B}, \kappa_{B}, 0\right)\right]^{3 / 2}}+\kappa_{\vec{r}_{-}, \vec{r}_{+}(1)} \frac{\partial A_{\vec{r}_{-}, \vec{r}_{+}(0)}}{\partial \kappa_{B}} \\
& =\frac{1}{2} \frac{f_{d}\left(\kappa_{B}, \kappa_{B}, r_{+}\right)+f_{d}\left(\kappa_{B}, \kappa_{B}, r_{-}\right)-\kappa_{\vec{r}_{-}, \vec{r}_{+}(1)} \frac{\partial}{\partial \kappa_{B}} f_{d}\left(\kappa_{B}, \kappa_{B}, 0\right)}{\left[f_{d}\left(\kappa_{B}, \kappa_{B}, 0\right)\right]^{3 / 2}} .
\end{aligned}
$$

Since the relative coordinate wavefunction is normalized,

$$
\begin{aligned}
& -\frac{1}{2 M}\left\langle\psi_{X}\left|\frac{\partial^{2}}{\partial X^{2}}\right| \psi_{X}\right\rangle \\
& =-\frac{\left(\frac{\partial A_{\vec{r}_{-}, \vec{r}_{+}}}{\partial X}\right)^{2}}{2 M A_{\vec{r}_{-}, \vec{r}_{+}}^{2}}+\frac{A_{\vec{r}_{-}, \vec{r}_{+}}^{2}}{2 M} \int_{x_{-} / 2}^{x_{+} / 2} d x_{d} \int d^{d-1} \vec{r}_{\perp}\left[\frac{\partial \phi_{\vec{r}_{-}, \vec{r}_{+}}}{\partial X}(\vec{r})\right]^{2} .
\end{aligned}
$$

We compute this term as an expansion in powers of $e^{-\kappa_{B} r_{+}}$and $e^{-\kappa_{B} r_{-}}$. At leading order we find 


$$
\begin{aligned}
& -\frac{1}{2 M}\left\langle\psi_{X}\left|\frac{\partial^{2}}{\partial X_{d}}\right| \psi_{X}\right\rangle_{(1)} \\
& =\frac{M}{2 m_{1}^{2} f_{d}\left(\kappa_{B}, \kappa_{B}, 0\right)} y_{d, 0}\left(\kappa_{B}, r_{+}\right)+\frac{M}{2 m_{2}^{2} f_{d}\left(\kappa_{B}, \kappa_{B}, 0\right)} y_{d, 0}\left(\kappa_{B}, r_{-}\right) .
\end{aligned}
$$

At second order

$$
\begin{aligned}
& -\frac{1}{2 M}\left\langle\psi_{X}\left|\frac{\partial^{2}}{\partial X^{2}}\right| \psi_{X}\right\rangle_{(2)} \\
& =T_{(A)}^{(2)}(X)+T_{(B)}^{(2)}(X)+T_{(C)}^{(2)}(X) \\
& +T_{(D 1)}^{(2)}(X)+T_{(D 2)}^{(2)}(X)+T_{(D 3)}^{(2)}(X)+T_{(D 4)}^{(2)}(X)+T_{(E)}^{(2)}(X),
\end{aligned}
$$

where the first term in Eq. (A11) is

$$
T_{(A)}^{(2)}(X)=-\frac{f_{d}\left(\kappa_{B}, \kappa_{B}, 0\right)}{2 M}\left(\frac{\partial A_{\vec{r}_{-}, \vec{r}_{+}(1)}}{\partial X}\right)^{2}+\cdots,
$$

and the second term

$$
\begin{aligned}
& T_{(B)}^{(2)}(X) \\
& =-\frac{M}{\mu^{2} f_{d}\left(\kappa_{B}, \kappa_{B}, 0\right)} y_{d, 0}\left(\kappa_{B}, r_{+}+r_{-}\right)+\frac{r_{+}+r_{-}}{\mu f_{d}\left(\kappa_{B}, \kappa_{B}, 0\right)} y_{d,-1}\left(\kappa_{B}, r_{+}+r_{-}\right),
\end{aligned}
$$

contains terms due to two derivatives with respect to the spatial coordinate without any contribution from the first-order corrections $A_{\vec{r}_{-}, \vec{r}_{+}(1)}$ and $\kappa_{\vec{r}_{-}, \vec{r}_{+}(1)}$.

$$
\begin{aligned}
T_{(C)}^{(2)}(X) & =\left.\frac{M \kappa_{\vec{r}_{-}, \vec{r}_{+}(1)}}{2 m_{1}^{2} f_{d}(\kappa, \kappa, 0)} \frac{\partial}{\partial \kappa}\left[y_{d, 0}\left(\kappa, r_{+}\right)\right]\right|_{\kappa=\kappa_{B}}+\left.\frac{M \kappa_{\vec{r}_{-}, \vec{r}_{+}(1)}}{2 m_{2}^{2} f_{d}(\kappa, \kappa, 0)} \frac{\partial}{\partial \kappa}\left[y_{d, 0}\left(\kappa, r_{-}\right)\right]\right|_{\kappa=\kappa_{B}} \\
& +\frac{A_{\vec{r}_{-}, \vec{r}_{+}(1)}}{\left[f_{d}\left(\kappa_{B}, \kappa_{B}, 0\right)\right]^{1 / 2}}\left[\frac{M}{m_{1}^{2}} y_{d, 0}\left(\kappa_{B}, r_{+}\right)+\frac{M}{m_{2}^{2}} y_{d, 0}\left(\kappa_{B}, r_{-}\right)\right]
\end{aligned}
$$

contains terms proportional to $\kappa_{\vec{r}_{-}, \vec{r}_{+}(1)}$ and $A_{\vec{r}_{-} \vec{r}_{+}(1)}$.

$$
\begin{aligned}
& T_{(D 1)}^{(2)}(X) \\
& \left.=-\frac{\kappa_{B}}{m_{1} f_{d}\left(\kappa_{B}, \kappa_{B}, 0\right)} \frac{\partial \kappa_{\vec{r}_{-}, \vec{r}_{+}(1)}^{\partial X}}{\partial X} \frac{r_{+}^{2}}{8} y_{d, 1}\left(\kappa_{B}, r_{+}\right)+\frac{r_{+}}{2} y_{d, 2}\left(\kappa_{B}, r_{+}\right)+\frac{3}{4} y_{d, 3}\left(\kappa_{B}, r_{+}\right)\right],
\end{aligned}
$$




$$
\begin{gathered}
T_{(D 2)}^{(2)}(X) \\
=-\frac{\kappa_{B}}{m_{2} f_{d}\left(\kappa_{B}, \kappa_{B}, 0\right)} \frac{\partial \kappa_{\vec{r}_{-}, \vec{r}_{+}(1)}}{\partial X}\left[\frac{r_{-}^{2}}{8} y_{d, 1}\left(\kappa_{B}, r_{-}\right)+\frac{r_{-}}{2} y_{d, 2}\left(\kappa_{B}, r_{-}\right)+\frac{3}{4} y_{d, 3}\left(\kappa_{B}, r_{-}\right)\right], \\
T_{(D 3)}^{(2)}(X)=\frac{\kappa_{B}}{4 m_{1} f_{d}\left(\kappa_{B}, \kappa_{B}, 0\right)} \frac{\partial \kappa_{\vec{r}_{-}, \vec{r}_{+}(1)}}{\partial X}\left[r_{+} y_{d, 2}\left(\kappa_{B}, r_{+}\right)+3 y_{d, 3}\left(\kappa_{B}, r_{+}\right)\right],
\end{gathered}
$$

and

$$
T_{(D 4)}^{(2)}(X)=\frac{\kappa_{B}}{4 m_{2} f_{d}\left(\kappa_{B}, \kappa_{B}, 0\right)} \frac{\partial \kappa_{\vec{r}_{-}, \vec{r}_{+}(1)}}{\partial X}\left[r_{-} y_{d, 2}\left(\kappa, r_{-}\right)+3 y_{d, 3}\left(\kappa, r_{-}\right)\right]
$$

contain terms due to a derivative with respect to $\kappa$ as well as a spatial derivative. Finally,

$$
\begin{aligned}
& T_{(E)}^{(2)}(X) \\
& =\frac{\left(\frac{\partial \kappa_{\vec{r}_{-}, \vec{r}_{+}(1)}}{\partial X}\right)^{2}}{24 M \kappa_{B}^{3} f_{d}\left(\kappa_{B}, \kappa_{B}, 0\right)}\left[-3 \frac{\partial y_{d, 0}}{\partial \kappa_{B}}\left(\kappa_{B}, 0\right)+3 \kappa \frac{\partial^{2} y_{d, 0}}{\partial \kappa_{B}^{2}}\left(\kappa_{B}, 0\right)-\kappa^{2} \frac{\partial^{3} y_{d, 0}}{\partial \kappa_{B}^{3}}\left(\kappa_{B}, 0\right)\right],
\end{aligned}
$$

contains terms due to two derivatives with respect to $\kappa$.

\section{Appendix B: Diagonal Adiabatic Correction in One Dimension}

Using Eq. A5 and Eq. A6 we compute the adiabatic correction in one spatial dimension. The first term in Eq. A11

$$
\begin{aligned}
& T_{(A)}^{(2)}(X)= \\
& -\frac{e^{-2 \kappa_{B} r_{+}} \kappa_{B}^{2}\left(m_{1}+m_{2}\right)\left[e^{\kappa_{B}\left(r_{+}+r_{-}\right)} m_{1}\left(3+\kappa_{B} r_{-}\right)+m_{2}\left(3-\kappa_{B} r_{+}\right)\right]^{2}}{2 m_{1}^{2} m_{2}^{2}},
\end{aligned}
$$

and the second term in Eq.A11, due to two spatial derivatives but not including contributions due to $A_{\vec{r}_{-}, \vec{r}_{+}(1)}$ and $\kappa_{\vec{r}_{-}, \vec{r}_{+}(1)}$, is

$$
\begin{aligned}
& T_{(B)}^{(2)}(X)= \\
& \frac{2 e^{-\kappa_{B}\left(r_{+}+r_{-}\right)} \kappa_{B}^{2}\left(m_{1}+m_{2}\right)\left(-m_{1}^{2}-m_{2}^{2}+m_{1} m_{2}\left[\kappa_{B} r_{+}+\kappa_{B} r_{-}-2\right]\right)}{m_{1}^{2} m_{2}^{2}} .
\end{aligned}
$$

The term proportional to $\kappa_{\vec{r}_{-}, \vec{r}_{+}(1)}$ and $A_{\vec{r}_{-}, \vec{r}_{+}(1)}$ is 


$$
\begin{aligned}
& T_{(C)}^{(2)}(X) \\
& =\frac{e^{-2 \kappa_{B} r_{-}}}{m_{2}^{2}} \kappa_{B}^{2}\left(m_{1}+m_{2}\right)\left(2 \kappa_{B} r_{-}-1\right)+\frac{e^{-2 \kappa_{B} r_{+}}}{m_{1}^{2}} \kappa_{B}^{2}\left(m_{1}+m_{2}\right)\left(2 \kappa_{B} r_{+}-1\right) \\
& +\frac{e^{-\kappa_{B}\left(r_{+}+r_{-}\right)}\left(m_{1}^{2}+m_{2}^{2}\right)}{m_{1}^{2} m_{2}^{2}} \kappa_{B}^{2}\left(m_{1}+m_{2}\right)\left(2 \kappa_{B} r_{+}+2 \kappa_{B} r_{-}-1\right) .
\end{aligned}
$$

The four terms due to one derivative with respect to $\kappa$ and one spatial derivative are

$$
\begin{aligned}
& T_{(D 1)}^{(2)}(X)= \\
& -\frac{\kappa_{B}^{2}\left(m_{1}+m_{2}\right)\left(6+4 \kappa_{B} r_{+}+\kappa_{B}^{2} r_{+}^{2}\right)}{2 m_{1}^{2} m_{2}^{2}}\left(m_{2} e^{-2 \kappa_{B} r_{+}}+m_{1} e^{-\kappa_{B}\left(r_{+}+r_{-}\right)}\right), \\
& T_{(D 2)}^{(2)}(X)= \\
& -\frac{\kappa_{B}^{2}\left(m_{1}+m_{2}\right)\left(9-4 \kappa_{B} r_{+}+\kappa_{B}^{2} r_{-}^{2}\right)}{2 m_{1} m_{2}^{2}}\left(m_{2} e^{-\kappa_{B}\left(r_{+}+r_{-}\right)}+m_{1} e^{-2 \kappa_{B} r_{-}}\right), \\
& T_{(D 3)}^{(2)}(X)=\frac{\kappa_{B}^{2}\left(m_{1}+m_{2}\right)\left(3+\kappa_{B} r_{+}\right)}{m_{1}^{2} m_{2}}\left(m_{2} e^{-2 \kappa_{B} r_{+}}+m_{1} e^{-\kappa_{B}\left(r_{+}+r_{-}\right)}\right),
\end{aligned}
$$

and

$$
T_{(D 4)}^{(2)}(X)=\frac{\kappa_{B}^{2}\left(m_{1}+m_{2}\right)\left(3+\kappa_{B} r_{-}\right)}{m_{1} m_{2}^{2}}\left(m_{2} e^{-\kappa_{B}\left(r_{+}+r_{-}\right)}+m_{1} e^{-2 \kappa_{B} r_{-}}\right) .
$$

The term containing contributions due to two derivatives with respect to $\kappa$ is

$$
T_{(E)}^{(2)}(X)=5 M \kappa_{B}^{2}\left(\frac{e^{-2 \kappa_{B} r_{+}}}{m_{1}^{2}}+\frac{e^{-2 \kappa_{B} r_{-}}}{m_{2}^{2}}\right)+\frac{10 e^{-\kappa_{B}\left(r_{+}+r_{-}\right)} \kappa_{B}^{2}}{\mu} .
$$

\section{Appendix C: Diagonal Adiabatic Correction in Two Dimensions}

Using Eq. A5 and Eq. A6 we compute the adiabatic correction in two spatial dimensions. We find that in two dimensions the first order correction to the binding momentum is

$$
\kappa_{\vec{r}_{-}, \vec{r}_{+}(1)}=-\kappa_{B}\left[K_{0}\left(\kappa_{B} r_{+}\right)+K_{0}\left(\kappa_{B} r_{-}\right)\right]
$$


the second order correction to the binding momentum is

$$
\begin{aligned}
\kappa_{\vec{r}_{-}, \vec{r}_{+}(2)} & =\frac{\kappa_{B}}{2}\left\{K_{0}\left(\kappa_{B} r_{+}\right)^{2}+K_{0}\left(\kappa_{B} r_{-}\right)^{2}+4 K_{0}\left[\kappa_{B}\left(r_{+}+r_{-}\right)\right]\right\} \\
& -\kappa_{B} K_{0}\left(\kappa_{B} r_{+}\right)\left[\kappa_{B} r_{+} K_{1}\left(\kappa_{B} r_{+}\right)+\kappa_{B} r_{-} K_{1}\left(\kappa_{B} r_{-}\right)-K_{0}\left(\kappa_{B} r_{-}\right)\right] \\
& -\kappa_{B}^{2} K_{0}\left(\kappa_{B} r_{-}\right)\left[r_{+} K_{1}\left(\kappa_{B} r_{+}\right)+\kappa_{B} r_{-} K_{1}\left(\kappa_{B} r_{-}\right)\right]
\end{aligned}
$$

and the generalized Yukawa function in two dimensions is

$$
y_{2,0}(\kappa, r)=\frac{1}{2 \pi} K_{0}(\kappa r)
$$

The first term in Eq. A11

$$
\begin{aligned}
& T_{(A)}^{(2)}(X) \\
& =-\frac{M \kappa_{B}^{2}}{8 m_{1}^{2} m_{2}^{2}}\left\{\kappa_{B} m_{1} r_{-} K_{0}\left(\kappa_{B} r_{-}\right)+\kappa_{B} m_{2} r_{+} K_{0}\left(\kappa_{B} r_{+}\right)-6 m_{1} K_{1}\left(\kappa_{B} r_{-}\right)\right. \\
& \left.-6 m_{2} K_{1}\left(\kappa_{B} r_{+}\right)+\kappa_{B} m_{1} r_{-} K_{2}\left(\kappa_{B} r_{-}\right)+\kappa_{B} m_{2} r_{+} K_{2}\left(\kappa_{B} r_{+}\right)\right\}^{2},
\end{aligned}
$$

and the second term due to two spatial derivatives but not including contributions due to $A_{\vec{r}_{-}, \vec{r}_{+}(1)}$ and $\kappa_{\vec{r}_{-}, \vec{r}_{+}(1)}$, is

$$
\begin{aligned}
& T_{(B)}^{(2)}(X) \\
& =-\frac{2 M \kappa_{B}^{2}}{m_{1}^{2} m_{2}^{2}}\left\{-\left(m_{1}+m_{2}\right)^{2} K_{0}\left[\kappa_{B}\left(r_{+}+r_{-}\right)\right]\right. \\
& \left.+\kappa_{B} m_{1} m_{2}\left(r_{+}+r_{-}\right) K_{1}\left[\kappa_{B}\left(r_{+}+r_{-}\right)\right]\right\} .
\end{aligned}
$$

The term proportional to $\kappa_{\vec{r}_{-}, \vec{r}_{+}(1)}$ and $A_{\vec{r}_{-}, \vec{r}_{+}(1)}$ is

$$
\begin{aligned}
T_{(C)}^{(2)}(X) & =-\left.\frac{\kappa^{3} M\left[K_{0}\left(\kappa r_{+}\right)+K_{0}\left(\kappa r_{-}\right)\right]}{m_{1}^{2}} \frac{\partial}{\partial \kappa}\left[K_{0}\left(\kappa r_{+}\right)\right]\right|_{\kappa=\kappa_{B}} \\
& +-\left.\frac{\kappa^{3} M\left[K_{0}\left(\kappa r_{+}\right)+K_{0}\left(\kappa r_{-}\right)\right]}{m_{2}^{2}} \frac{\partial}{\partial \kappa}\left[K_{0}\left(\kappa r_{-}\right)\right]\right|_{\kappa=\kappa_{B}} \\
& +2 \kappa \sqrt{\pi} A_{\vec{r}_{-}, \vec{r}_{+}(1)}\left[\frac{M}{2 \pi m_{1}^{2}} K_{0}\left(\kappa_{B} r_{+}\right)+\frac{M}{2 \pi m_{2}^{2}} K_{0}\left(\kappa_{B} r_{-}\right)\right]
\end{aligned}
$$

where 


$$
A_{\vec{r}_{-}, \vec{r}_{+}(1)}=\sqrt{\pi} \kappa_{B}\left[-2 K_{0}\left(\kappa_{B} r_{-}\right)-2 K_{0}\left(\kappa_{B} r_{+}\right)+\kappa_{B} r_{-} K_{1}\left(\kappa_{B} r_{-}\right)+\kappa_{B} r_{+} K_{1}\left(\kappa_{B} r_{+}\right)\right]^{-1 / 2}
$$

The four terms due to one derivative with respect to $\kappa$ and one spatial derivative are

$$
\begin{aligned}
& T_{(D 1)}^{(2)}(X) \\
& =-\frac{M \kappa_{B}^{2}}{4 m_{1}^{2} m_{2}}\left[m_{1} K_{1}\left(\kappa_{B} r_{-}\right)+m_{2} K_{1}\left(\kappa_{B} r_{+}\right)\right]\left\{-3 \kappa_{B}^{2} r_{+}^{2} G_{1,3}^{2,1}\left[\frac{\kappa_{B} r_{+}}{2}, \frac{1}{2} \mid \begin{array}{c}
0 \\
-\frac{1}{2}, \frac{1}{2},-1
\end{array}\right]\right. \\
& \left.+3 \pi\left[\kappa_{B} r_{+} K_{0}\left(\kappa_{B} r_{+}\right)+2 K_{1}\left(\kappa_{B} r_{+}\right)\right] L_{1}\left(\kappa_{B} r_{+}\right)+\kappa_{B} r_{+} K_{1}\left(\kappa_{B} r_{+}\right)\left[4 \kappa_{B} r_{+}+3 \pi L_{2}\left(\kappa_{B} r_{+}\right)\right]\right\},
\end{aligned}
$$

where $L_{\alpha}$ is the modified Stuve function and $G_{p, q}^{m, n}$ is the generalized Meijer G-function.

$$
\begin{aligned}
& T_{(D 2)}^{(2)}(X) \\
& =-\frac{M \kappa_{B}^{2}}{4 m_{1} m_{2}^{2}}\left[m_{1} K_{1}\left(\kappa_{B} r_{-}\right)+m_{2} K_{1}\left(\kappa_{B} r_{+}\right)\right]\left\{-3 \kappa_{B}^{2} r_{-}^{2} G_{1,3}^{2,1}\left[\frac{\kappa_{B} r_{-}}{2}, \frac{1}{2} \mid \begin{array}{c}
0 \\
-\frac{1}{2}, \frac{1}{2},-1
\end{array}\right]\right. \\
& \left.+3 \pi\left[\kappa_{B} r_{-} K_{0}\left(\kappa_{B} r_{-}\right)+2 K_{1}\left(\kappa_{B} r_{-}\right)\right] L_{1}\left(\kappa_{B} r_{-}\right)+\kappa_{B} r_{-} K_{1}\left(\kappa_{B} r_{-}\right)\left[4 \kappa_{B} r_{-}+3 \pi L_{2}\left(\kappa_{B} r_{-}\right)\right]\right\} . \\
& T_{(D 3)}^{(2)}(X)=\frac{\pi \kappa_{B}^{2}}{m_{1}} \frac{\partial \kappa_{\vec{r}_{-}, \vec{r}_{+}(1)}}{\partial X}\left[r_{+} y_{2,2}\left(\kappa_{B}, r_{+}\right)+3 y_{2,3}\left(\kappa_{B}, r_{+}\right)\right],
\end{aligned}
$$

where the spatial derivative of the first order correction to the binding momentum is

$$
\frac{\partial \kappa_{\vec{r}_{-}, \vec{r}_{+}(1)}}{\partial X}=\frac{2 M}{m_{1}} \kappa_{B}^{2} K_{1}\left(\kappa_{B} r_{+}\right)+\frac{2 M}{m_{2}} \kappa_{B}^{2} K_{1}\left(\kappa_{B} r_{-}\right) .
$$

The generalized Yukawa function $y_{2,2}(\kappa, r)$ is

$$
y_{2,2}(\kappa, r)=-\frac{r^{2}}{4}\left[\frac{1}{\kappa r}-K_{0}(\kappa r) L_{-1}(\kappa r)-K_{1}(\kappa r) L_{0}(\kappa r)\right]+\frac{r}{2 \pi \kappa} K_{1}(\kappa r)
$$


the generalized Yukawa function $y_{2,3}(\kappa, r)$ is

$$
\begin{aligned}
y_{2,3}(\kappa, r) & =\frac{r^{3}}{8}\left[\frac{1}{\kappa r}-K_{0}(\kappa r) L_{-1}(\kappa r)-K_{1}(\kappa r) L_{0}(\kappa r)\right] \\
& +\frac{r}{8 \kappa^{2}} K_{0}(\kappa r) L_{1}(\kappa r) \\
& +\frac{1}{24 \pi \kappa^{3}} K_{1}(\kappa r)\left[-4 \kappa^{2} r^{2}+6 \pi L_{1}(\kappa r)+3 \pi \kappa r L_{2}(\kappa r)\right] \\
& -\frac{r^{2}}{8 \pi \kappa} G_{1,3}^{2,1}\left[\frac{\kappa r}{2}, \frac{1}{2} \mid \begin{array}{c}
0 \\
-\frac{1}{2}, \frac{1}{2},-1
\end{array}\right],
\end{aligned}
$$

and

$$
T_{(D 4)}^{(2)}(X)=\frac{\pi \kappa_{B}^{2}}{m_{2}} \frac{\partial \kappa_{\vec{r}_{-}, \vec{r}_{+}(1)}}{\partial X}\left[r_{-} y_{2,2}\left(\kappa, r_{-}\right)+3 y_{2,3}\left(\kappa, r_{-}\right)\right] .
$$

The term containing contributions due to two derivatives with respect to $\kappa$ is

$$
T_{(E)}^{(2)}(X)=\frac{8 \kappa_{B}^{2}\left(m_{1}+m_{2}\right)\left[m_{1} K_{1}\left(\kappa_{B} r_{-}\right)+m_{2} K_{1}\left(\kappa_{B} r_{+}\right)\right]^{2}}{3 m_{1}^{2} m_{2}^{2}} .
$$

\section{Appendix D: Diagonal Adiabatic Correction in Three Dimensions}

For the effective Hamiltonian in three dimensions $\kappa_{\vec{r}_{-}, \vec{r}_{+}(1)}, \kappa_{\vec{r}_{-}, \vec{r}_{+}(2)}, T_{(A)}^{(2)}(X), T_{(B)}^{(2)}(X)$, $T_{(C)}^{(2)}(X), T_{(D 1-D 4)}^{(2)}(X)$, and $T_{(E)}^{(2)}(X)$ are defined as follows. The first order correction to the binding momentum is

$$
\kappa_{\vec{r}_{-}, \vec{r}_{+}(1)}=\frac{y_{3,0}\left(\kappa_{B}, r_{+}\right)+y_{3,0}\left(\kappa_{B}, r_{-}\right)}{\frac{\partial}{\partial \kappa_{B}} y_{3,0}\left(\kappa_{B}, 0\right)}
$$

the second order correction to the binding momentum is

$$
\begin{aligned}
& \kappa_{\vec{r}_{-}, \vec{r}_{+}(2)} \\
& =\frac{1}{\frac{\partial}{\partial \kappa_{B}} y_{3,0}\left(\kappa_{B}, 0\right)}\left\{\frac{y_{3,0}\left(\kappa_{B}, r_{+}\right)+y_{3,0}\left(\kappa_{B}, r_{-}\right)}{\frac{\partial}{\partial \kappa_{B}} y_{3,0}\left(\kappa_{B}, 0\right)} \frac{\partial}{\partial \kappa_{B}}\left[y_{3,0}\left(\kappa_{B}, r_{+}\right)+y_{3,0}\left(\kappa_{B}, r_{-}\right)\right]\right. \\
& \left.-2 y_{3,0}\left(\kappa_{B}, r_{+}+r_{-}\right)-\frac{\left[y_{3,0}\left(\kappa_{B}, r_{+}\right)+y_{3,0}\left(\kappa_{B}, r_{-}\right)\right]^{2}}{2\left[\frac{\partial}{\partial \kappa_{B}} y_{3,0}\left(\kappa_{B}, 0\right)\right]^{2}} \frac{\partial^{2}}{\partial \kappa_{B}^{2}} y_{3,0}\left(\kappa_{B}, 0\right)\right\},
\end{aligned}
$$


and the generalized Yukawa function in three spatial dimensions is

$$
y_{3,0}(\kappa, r)=\frac{e^{-\kappa r}}{4 \pi r} \text {. }
$$

The first term in Eq. A11 is

$$
\begin{aligned}
& T_{(A)}^{(2)}(X) \\
& =-\frac{f_{3}\left(\kappa_{B}, \kappa_{B}, 0\right)}{2 M}\left(\frac{\partial A_{\vec{r}_{-}, \vec{r}_{+}(1)}}{\partial X}\right)^{2},
\end{aligned}
$$

where the overlap integral in three spatial dimensions is

$$
f_{3}(\kappa, \kappa, r)=\frac{e^{-\kappa r}}{8 \pi \kappa}
$$

and the spatial derivative of the first order correction to the wavefunction normalization is

$$
\begin{aligned}
& \frac{\partial A_{\vec{r}_{-}, \vec{r}_{+}(1)}}{\partial X} \\
& =\frac{1}{2} \frac{\frac{2 M}{m_{1}} \frac{\partial}{\partial r_{+}} f_{3}\left(\kappa_{B}, \kappa_{B}, r_{+}\right)+\frac{2 M}{m_{2}} \frac{\partial}{\partial r_{-}} f_{3}\left(\kappa_{B}, \kappa_{B}, r_{-}\right)-\frac{\partial \kappa_{(1)}}{\partial X} \frac{\partial}{\partial \kappa_{B}} f_{3}\left(\kappa_{B}, \kappa_{B}, 0\right)}{\left[f_{3}\left(\kappa_{B}, \kappa_{B}, 0\right)\right]^{3 / 2}} .
\end{aligned}
$$

The next term, with contributions due solely to two spatial derivatives, is

$$
\begin{aligned}
& T_{(B)}^{(2)}(X) \\
& =-\frac{M}{\mu^{2} f_{3}\left(\kappa_{B}, \kappa_{B}, 0\right)} y_{3,0}\left(\kappa_{B}, r_{+}+r_{-}\right)+\frac{r_{+}+r_{-}}{\mu f_{3}\left(\kappa_{B}, \kappa_{B}, 0\right)} y_{3,-1}\left(\kappa_{B}, r_{+}+r_{-}\right),
\end{aligned}
$$

where the generalized Yukawa function, $y_{3,-1}(\kappa, r)$, is

$$
y_{3,-1}(\kappa, r)=-\frac{\partial}{\partial \kappa_{B}} y_{3,0}\left(\kappa_{B}, r\right) .
$$

The term containing contributions due to $\kappa_{\vec{r}_{-}, \vec{r}_{+}(1)}$ and $A_{\vec{r}_{-}, \vec{r}_{+}(1)}$ is

$$
\begin{aligned}
& T_{(C)}^{(2)}(X) \\
& =\left.\frac{M \kappa_{\vec{r}_{-}, \vec{r}_{+}(1)}^{2}}{2 m_{1}^{2} f_{3}(\kappa, \kappa, 0)} \frac{\partial}{\partial \kappa}\left[y_{3,0}\left(\kappa, r_{+}\right)\right]\right|_{\kappa=\kappa_{B}} \\
& +\left.\frac{M \kappa_{\vec{r}_{-}, \vec{r}_{+}(1)}}{2 m_{2}^{2} f_{3}(\kappa, \kappa, 0)} \frac{\partial}{\partial \kappa}\left[y_{3,0}\left(\kappa, r_{-}\right)\right]\right|_{\kappa=\kappa_{B}} \\
& +\frac{A_{\vec{r}_{-}, \vec{r}_{+}(1)}}{\left[f_{3}\left(\kappa_{B}, \kappa_{B}, 0\right)\right]^{1 / 2}}\left[\frac{M}{m_{1}^{2}} y_{3,0}\left(\kappa_{B}, r_{+}\right)+\frac{M}{m_{2}^{2}} y_{3,0}\left(\kappa_{B}, r_{-}\right)\right] .
\end{aligned}
$$


The four terms that result from taking one derivative with respect to $\kappa$ and one spatial derivative are

$$
\begin{aligned}
& T_{(D 1)}^{(2)}(X) \\
& =-\frac{\kappa_{B}}{m_{1} f_{3}\left(\kappa_{B}, \kappa_{B}, 0\right)} \frac{\partial \kappa_{\vec{r}_{-}, \vec{r}_{+}(1)}}{\partial X}\left[\frac{r_{+}^{2}}{8} y_{3,1}\left(\kappa_{B}, r_{+}\right)+\frac{r_{+}}{2} y_{3,2}\left(\kappa_{B}, r_{+}\right)+\frac{3}{4} y_{3,3}\left(\kappa_{B}, r_{+}\right)\right]
\end{aligned}
$$

where the spatial derivative of the first order correction to $\kappa_{\vec{r}_{-}, \vec{r}_{+}}$is

$$
\frac{\partial \kappa_{\vec{r}_{-}, \vec{r}_{+}(1)}}{\partial X}=\frac{2 M\left[\frac{1}{m_{1}} \frac{\partial}{\partial r_{+}} y_{3,0}\left(\kappa_{B}, r_{+}\right)+\frac{1}{m_{2}} \frac{\partial}{\partial r_{+}} y_{3,0}\left(\kappa_{B}, r_{-}\right)\right]}{\frac{\partial}{\partial \kappa_{B}} y_{3,0}\left(\kappa_{B}, 0\right)}
$$

and the generalized Yukawa functions, $y_{3,1}(\kappa, r), y_{3,2}(\kappa, r)$, and $y_{3,3}(\kappa, r)$, are

$$
\begin{gathered}
y_{3,1}(\kappa, r)=-\frac{1}{4 \pi} \operatorname{Ei}(-\kappa r), \\
y_{3,2}(\kappa, r)=\frac{e^{-\kappa r}}{4 \pi \kappa}+\frac{r}{4 \pi} \operatorname{Ei}(-\kappa r),
\end{gathered}
$$

and

$$
y_{3,3}(\kappa, r)=\frac{e^{-\kappa r}}{8 \pi \kappa^{2}}(1-\kappa r)-\frac{r^{2}}{8 \pi} \operatorname{Ei}(-\kappa r)
$$

Here Ei is the exponential integral function.

$$
\begin{aligned}
& T_{(D 2)}^{(2)}(X) \\
& =-\frac{\kappa_{B}}{m_{2} f_{3}\left(\kappa_{B}, \kappa_{B}, 0\right)} \frac{\partial \kappa_{\vec{r}_{-}, \vec{r}_{+}(1)}}{\partial X}\left[\frac{r_{-}^{2}}{8} y_{3,1}\left(\kappa_{B}, r_{-}\right)+\frac{r_{-}}{2} y_{3,2}\left(\kappa_{B}, r_{-}\right)+\frac{3}{4} y_{3,3}\left(\kappa_{B}, r_{-}\right)\right] \\
& T_{(D 3)}^{(2)}(X)=\frac{\kappa_{B}}{4 m_{1} f_{3}\left(\kappa_{B}, \kappa_{B}, 0\right)} \frac{\partial \kappa_{\vec{r}_{-}, \vec{r}_{+}(1)}}{\partial X}\left[r_{+} y_{3,2}\left(\kappa_{B}, r_{+}\right)+3 y_{3,3}\left(\kappa_{B}, r_{+}\right)\right]
\end{aligned}
$$

and

$$
T_{(D 4)}^{(2)}(X)=\frac{\kappa_{B}}{4 m_{2} f_{3}\left(\kappa_{B}, \kappa_{B}, 0\right)} \frac{\partial \kappa_{\vec{r}_{-}, \vec{r}_{+}(1)}}{\partial X}\left[r_{-} y_{3,2}\left(\kappa, r_{-}\right)+3 y_{3,3}\left(\kappa, r_{-}\right)\right]
$$

The term containing contributions due to two derivatives with respect to $\kappa$ is

$$
T_{(E)}^{(2)}(X)=\frac{\left(\frac{\partial \kappa_{\vec{r}_{-}, \vec{r}_{+}(1)}}{\partial X}\right)^{2}}{24 M \kappa_{B}^{3} f_{3}\left(\kappa_{B}, \kappa_{B}, 0\right)}\left[-3 \frac{\partial y_{3,0}}{\partial \kappa_{B}}\left(\kappa_{B}, 0\right)+3 \kappa \frac{\partial^{2} y_{3,0}}{\partial \kappa_{B}^{2}}\left(\kappa_{B}, 0\right)-\kappa^{2} \frac{\partial^{3} y_{3,0}}{\partial \kappa_{B}^{3}}\left(\kappa_{B}, 0\right)\right]
$$




\section{Appendix E: Summary of Continuum Extrapolations}

In one dimension for mass ratio $m_{2} / m_{1}=1$ we consider $L_{z}|c|=8,10,20$, and for $L_{z}|c|=$ 10 we consider the ground state as well as the first and second excited states. Table $\bigvee$ shows a summary of the continuum extrapolations in one dimension for mass ratio $m_{2} / m_{1}=1$.

\begin{tabular}{|c|c|c|c|}
$L_{z}|c|$ & $L_{z}$ & $R \kappa_{B}$ & $E_{K} /\left|E_{B}\right|$ \\
\hline \hline 20 & $200,300,400$ & 0.498 & 0.006832 \\
10 & $100,200,300,400$ & 0.4950 & 0.030 \\
8 & $80,160,240$ & 0.4981 & 0.050122 \\
10 & $100,200,300$ & 0.4812 & 0.12083 \\
10 & $100,200,300$ & 0.461 & 0.2694
\end{tabular}

TABLE V: Summary of continuum extrapolations for $m_{2} / m_{1}=1$ in one dimension

Table VI gives the values of $L_{z}|c|$ and $L_{z}$ considered as well as the continuum limit extrapolations in one dimension for mass ratio $m_{2} / m_{1}=2$.

\begin{tabular}{|c|c|c|c|}
$L_{z}|c|$ & $L_{z}$ & $R \kappa_{B}$ & $E_{K} /\left|E_{B}\right|$ \\
\hline \hline 20 & $200,300,400$ & 0.6041 & 0.0062 \\
10 & $100,200,300$ & 0.5957 & 0.028228 \\
8 & $80,160,240$ & 0.5885 & 0.0471 \\
10 & $100,200,300$ & 0.5676 & 0.111164 \\
10 & $100,200,300$ & 0.5311 & 0.247
\end{tabular}

TABLE VI: Summary of continuum extrapolations for $m_{2} / m_{1}=2$ in one dimension

In one dimension for mass ratio $m_{2} / m_{1}=4$ the continuum extrapolations are given by Table VII. 


\begin{tabular}{|c|c|c|c|}
$L_{z}|c|$ & $L_{z}$ & $R \kappa_{B}$ & $E_{K} /\left|E_{B}\right|$ \\
\hline \hline 20 & $200,300,400$ & 0.8694 & 0.00473 \\
10 & $100,200,300$ & 0.8504 & 0.02292 \\
8 & $80,160,240$ & 0.8343 & 0.039 \\
10 & $100,200,300$ & 0.7915 & 0.0891 \\
10 & $100,200,300$ & 0.7214 & 0.19409
\end{tabular}

TABLE VII: Summary of continuum extrapolations for $m_{2} / m_{1}=4$ in one dimension

In one dimension for mass ratio $m_{2} / m_{1}=8$ we get the continuum extrapolations as shown in Table VIII.

\begin{tabular}{|c|c|c|c|}
$L_{z}|c|$ & $L_{z}$ & $R \kappa_{B}$ & $E_{K} /\left|E_{B}\right|$ \\
\hline \hline 20 & $200,300,400$ & 1.206 & 0.003151 \\
10 & $100,200,300$ & 1.1742 & 0.01665 \\
8 & $80,160,240$ & 1.1455 & 0.029907 \\
10 & $100,200,300$ & 1.083 & 0.0635 \\
10 & $100,200,300$ & 0.982 & 0.13589
\end{tabular}

TABLE VIII: Summary of continuum extrapolations for $m_{2} / m_{1}=8$ in one dimension

In two dimensions for mass ratios $m_{2} / m_{1}=1,2,4,8$ we consider the ground and first excited state for each $L_{z} \kappa_{B}=6.8,10.2$. As noted previously $L_{z}$ is the length of the lattice in the direction perpendicular to the confining wall. Table IX shows a summary of the continuum extrapolations in two dimensions for mass ratio $m_{2} / m_{1}=1$.

\begin{tabular}{|c|c|c|c|}
$L_{z} \kappa_{B}$ & $L_{z}$ & $R \kappa_{B}$ & $E_{K} /\left|E_{B}\right|$ \\
\hline \hline 10.2 & $60,90,120,150$ & 0.485146 & 0.004742 \\
6.8 & $40,60,80,100$ & 0.456299 & 0.011126 \\
10.2 & $60,90,120,150$ & 0.482144 & 0.019341 \\
6.8 & $40,60,80,100$ & 0.45136 & 0.044811
\end{tabular}

TABLE IX: Summary of continuum extrapolations for $m_{2} / m_{1}=1$ in two dimensions

Table $\mathrm{X}$ gives the values of $L_{z} \kappa_{B}$ and $L_{z}$ considered as well as the continuum limit extrapolations in two dimensions for mass ratio $m_{2} / m_{1}=2$. 


\begin{tabular}{|c|c|c|c|}
$L_{z} \kappa_{B}$ & $L_{z}$ & $R \kappa_{B}$ & $E_{K} /\left|E_{B}\right|$ \\
\hline \hline 10.2 & $60,90,120,150$ & 0.572157 & 0.004342 \\
6.8 & $40,60,80,100$ & 0.541738 & 0.010386 \\
10.2 & $60,90,120,150$ & 0.567111 & 0.017369 \\
6.8 & $40,60,80,100$ & 0.532998 & 0.040117
\end{tabular}

TABLE X: Summary of continuum extrapolations for $m_{2} / m_{1}=2$ in two dimensions

In two dimensions for mass ratio $m_{2} / m_{1}=4$ the continuum extrapolations are given by Table XI.

\begin{tabular}{|c|c|c|c|}
$L_{z} \kappa_{B}$ & $L_{z}$ & $R \kappa_{B}$ & $E_{K} /\left|E_{B}\right|$ \\
\hline \hline 10.2 & $60,90,120,150$ & 0.791197 & 0.003473 \\
6.8 & $40,60,80,100$ & 0.759959 & 0.007583 \\
10.2 & $60,90,120,150$ & 0.783424 & 0.013027 \\
6.8 & $40,60,80,100$ & 0.742787 & 0.031096
\end{tabular}

TABLE XI: Summary of continuum extrapolations for $m_{2} / m_{1}=4$ in two dimensions

In two dimensions for mass ratio $m_{2} / m_{1}=8$ we get the continuum extrapolations as shown in Table XII.

\begin{tabular}{|c|c|c|c|}
$L_{z} \kappa_{B}$ & $L_{z}$ & $R \kappa_{B}$ & $E_{K} /\left|E_{B}\right|$ \\
\hline \hline 10.2 & $60,90,120,150$ & 1.81456 & 0.09496 \\
6.8 & $40,60,80,100$ & 1.1018 & 0.17597 \\
10.2 & $60,90,120,150$ & 1.21421 & 0.20091 \\
6.8 & $40,60,80,100$ & 0.79017 & 0.43994
\end{tabular}

TABLE XII: Summary of continuum extrapolations for $m_{2} / m_{1}=8$ in two dimensions

In three dimensions we consider the ground and first excited states of $L_{z} \kappa_{B}=4.9,6$. Table XIII shows a summary of the continuum extrapolations in three dimensions for mass ratio $m_{2} / m_{1}=1$

Table XIV] gives the values of $L_{z} \kappa_{B}$ and $L_{z}$ considered as well as the continuum limit extrapolations in three dimensions for mass ratio $m_{2} / m_{1}=2$. 


\begin{tabular}{|c|c|c|c|}
$L_{z} \kappa_{B}$ & $L_{z}$ & $R \kappa_{B}$ & $E_{K} /\left|E_{B}\right|$ \\
\hline \hline 6 & $24,36,48$ & 0.27263 & 0.08294 \\
4.9 & $20,30,40$ & 0.26891 & 0.12394 \\
6 & $24,36,48$ & 0.25605 & 0.32787 \\
4.94 & $20,30,40$ & 0.24773 & 0.48669
\end{tabular}

TABLE XIII: Summary of continuum extrapolations for $m_{2} / m_{1}=1$ in three dimensions

\begin{tabular}{|c|c|c|c|}
$L_{z} \kappa_{B}$ & $L_{z}$ & $R \kappa_{B}$ & $E_{K} /\left|E_{B}\right|$ \\
\hline \hline 6 & $24,36,48$ & 0.32937 & 0.0769 \\
4.9 & $20,30,40$ & 0.32308 & 0.11573 \\
6 & $24,36,48$ & 0.30134 & 0.30132 \\
4.9 & $20,30,40$ & 0.28801 & 0.44853
\end{tabular}

TABLE XIV: Summary of continuum extrapolations for $m_{2} / m_{1}=2$ in three dimensions

In three dimensions for mass ratio $m_{2} / m_{1}=4$ the continuum extrapolations are given by Table XV.

\begin{tabular}{|c|c|c|c|}
$L_{z} \kappa_{B}$ & $L_{z}$ & $R \kappa_{B}$ & $E_{K} /\left|E_{B}\right|$ \\
\hline \hline 6 & $24,36,48$ & 0.47906 & 0.06215 \\
4.9 & $20,30,40$ & 0.46579 & 0.09545 \\
6 & $24,36,48$ & 0.42392 & 0.23812 \\
4.9 & $20,30,40$ & 0.39833 & 0.35779
\end{tabular}

TABLE XV: Summary of continuum extrapolations for $m_{2} / m_{1}=4$ in three dimensions

In three dimensions for mass ratio $m_{2} / m_{1}=8$ we get the continuum extrapolations as shown in Table XVI. 


\begin{tabular}{|c|c|c|c|}
$L_{z} \kappa_{B}$ & $L_{z}$ & $R \kappa_{B}$ & $E_{K} /\left|E_{B}\right|$ \\
\hline \hline 6 & $24,36,48$ & 0.68252 & 0.0454 \\
4.9 & $20,30,40$ & 0.65848 & 0.07191 \\
6 & $24,36,48$ & 0.59503 & 0.16868 \\
4.9 & $20,30,40$ & 0.55407 & 0.25769
\end{tabular}

TABLE XVI: Summary of continuum extrapolations for $m_{2} / m_{1}=8$ in three dimensions 